\title{
AGE-RELATED CHANGES IN OVERCOMING PROACTIVE INTERFERENCE IN ASSOCIATIVE MEMORY: THE ROLE OF PFC-MEDIATED EXECUTIVE CONTROL PROCESSES AT RETRIEVAL
}

Michael R. Dulas and Audrey Duarte

Georgia Institute of Technology

Address:

School of Psychology

Georgia Institute of Technology

654 Cherry Street, Atlanta, GA

30332-0170

Correspondence to:

Michael R. Dulas, Ph.D.

Beckman Institute

405 N Mathews Ave

Urbana, IL 61801

mrdulas@illinois.edu

Phone +1 5857320276 


\begin{abstract}
Behavioral evidence has shown age-related impairments in overcoming proactive interference in memory, but it is unclear what underlies this deficit. Imaging studies in the young suggest overcoming interference may require several executive control processes supported by the ventrolateral prefrontal cortex (VLPFC) and dorsolateral PFC (DLPFC). The present functional magnetic resonance imaging (fMRI) study investigated whether age-related changes in dissociable executive control processes underlie deficits in overcoming proactive interference in associative memory during retrieval. Participants were tasked with remembering which associate (face or scene) objects were paired with most recently during study, under conditions of high or low proactive interference. Behavioral results demonstrated that, as interference increased, memory performance decreased similarly across groups, with slight associative memory deficits in older adults. Imaging results demonstrated that, across groups, left midVLPFC showed increasing activity with increasing interference, though activity did not distinguish correct from incorrect associative memory responses, suggesting this region may not directly serve in successful resolution of proactive interference, per se. Under conditions of high interference, older adults showed reduced associative memory accuracy effects in the DLPFC and anterior PFC. These results suggest that age-related PFC dysfunction may not be ubiquitous. Executive processes supported by ventral regions that detect mnemonic interference may be less affected than processes supported by dorsal and anterior regions that directly resolve interference.
\end{abstract}

Keywords: Aging, Associative Memory, Executive Control, fMRI, Prefrontal Cortex 


\section{INTRODUCTION}

Behavioral evidence has suggested that older adults may be particularly impaired on memory tasks that require higher levels of recruitment of executive functions (Hasher \& Zacks, 1979; Johnson, Hashtroudi, \& Lindsay, 1993). Such tasks may include associative memory tasks in which participants must overcome proactive interference. Proactive interference is induced when previous, but no longer valid, information conflicts with current task goals. For example, difficulty remembering a friend's new email address may stem from a strong memory for their previous, but no longer valid, email address. Several studies have shown that older adults demonstrate larger susceptibility to proactive interference compared to young adults in both working memory and episodic memory tasks (Bowles \& Salthouse, 2003; Jacoby, Bishara, Hessels, \& Toth, 2005; Jonides et al., 2000; see Lustig \& Jantz, 2015 for review; Lustig, May, \& Hasher, 2001; May, Hasher, \& Kane, 1999). The neurocognitive mechanisms underlying this increased susceptibility to proactive interference in older adults are largely unknown. We will investigate these processes in the current study.

Recent neuroimaging evidence suggests that the ventrolateral prefrontal cortex (VLPFC) (BAs 44, 45, and 47) (Jonides \& Nee, 2006; Nee, Wager, \& Jonides, 2007; Oztekin, Curtis, \& McElree, 2009), and more specifically, the left mid-VLPFC (BA 44/45) (Badre, Poldrack, PareBlagoev, Insler, \& Wagner, 2005; Badre \& Wagner, 2007), is particularly involved in overcoming proactive interference. Further, both lesion (Thompson-Schill et al., 2002) and transcranial magnetic stimulation studies (TMS) (Feredoes, Heinen, Weiskopf, Ruff, \& Driver, 2011) have demonstrated that disruption of the left VLPFC results in both increased errors and increased response times in response to proactive interference in working memory tasks. It has been suggested that left mid-VLPFC supports a process referred to as post-retrieval selection, 
which is thought to resolve competition between multiple active representations (Badre et al., 2005; Badre \& Wagner, 2007) and is suggested to be critical to the successful resolution of proactive interference (see Badre \& Wagner, 2007 for review). That is, after information is retrieved, irrelevant/conflicting information must be suppressed and relevant information likely enhanced. As with the email example, when someone attempts to retrieve their friend's new email, he/she may retrieve both the old and new email addresses. To make a correct memory decision, the person must inhibit the no longer valid, but perhaps strongly encoded previous email address, and select the new, currently relevant email address. Behavioral evidence suggests that post-retrieval selection requires additional processing time, as correct responses to high interference trials are significantly slower than for low interference trials (Jonides \& Nee, 2006). One cause of older adults' associative memory impairments may be that they are less likely to engage this process, and thus may incorrectly select the more salient, but no longer valid, associate. It should be noted that left mid-VLPFC is recruited with increasing selection demands across a variety of tasks (see Badre, 2008; Nelson, Reuter-Lorenz, Persson, Sylvester, \& Jonides, 2009 for reviews), including lexical decision-making (Gold et al., 2006) and semantic interference tasks involving competition via schematically related items (e.g. rejecting the word SLEEP when presented with BED, PILLOW, TIRED, NIGHT) (Atkins \& Reuter-Lorenz, 2011). This suggests that it is engaged whenever resolution of conceptual (as opposed to motor) interference is necessary (Badre \& Wagner, 2007).

Although the left VLPFC is repeatedly implicated in tasks involving proactive and semantic interference, recent research has called into question whether the left VLPFC is directly involved in this selection process, in which competition in memory is successfully resolved (Atkins \& Reuter-Lorenz, 2011; Han, O'Connor, Eslick, \& Dobbins, 2012; Oztekin et al., 2009). 
That is, while this region has been repeatedly implicated in detecting mnemonic interference, many of these studies have only analyzed correct response trials, and thus have not investigated whether this region directly supports correct resolution of interference. Alternatively, the left VLPFC may be sensitive to mnemonic interference but may not support accuracy, per se. To our knowledge, only two previous working memory functional magnetic resonance imaging (fMRI) studies have looked at this issue (Atkins \& Reuter-Lorenz, 2011; Oztekin et al., 2009). In both studies, the left VLPFC showed greater activity for high interference compared to low interference trials, but did not dissociate successful from unsuccessful responses. Thus, while the left VLPFC may play a role in detection of and attempts to resolve interference between competing mnemonic representations, it may not directly contribute to successful interference resolution.

While the left VLPFC did not distinguish successful vs. unsuccessful resolution of interference, both studies showed evidence of other regions that did, including the dorsolateral prefrontal cortex (DLPFC) (Atkins \& Reuter-Lorenz, 2011). In many episodic memory studies, the DLPFC has been implicated in a process referred to as post-retrieval monitoring (Achim \& Lepage, 2005; Donaldson, Wheeler, \& Petersen, 2010; Dulas \& Duarte, 2012; Henson, Shallice, \& Dolan, 1999; Ranganath, Heller, \& Wilding, 2007; Turner, Simons, Gilbert, Frith, \& Burgess, 2008). Post-retrieval monitoring refers to the evaluation and manipulation of the products of retrieval (Henson, Shallice, et al., 1999). Previous evidence has shown that post-retrieval monitoring effects are greater when one is near his/her decision criterion (Henson, Rugg, Shallice, \& Dolan, 2000). If demands on post-retrieval monitoring are greater when one is near his/her decision criterion, it is conceivable that the DLPFC may be sensitive to memory accuracy under conditions of high interference. Another related view of the DLPFC is that it contributes to 
post-retrieval decision-making, in that activity increases with the number of decisions that need to be made after a retrieval attempt (Dobbins \& Han, 2006; Han, Huettel, \& Dobbins, 2009). Under this view, correct high interference responses also may require more internal decisions (e.g. "Did I see both of these items previously? Which item was most recent?" etc.) than an incorrect response, wherein the more salient but no longer valid associate is selected.

The frontal aging hypothesis suggests that aging leads to disproportionate alterations in PFC-mediated executive control processes (West, 1996). There has been little research, however, investigating the effects of age on separable components of executive control. As stated previously, behavioral evidence has consistently demonstrated that older adults show deficits on episodic memory tasks involving proactive interference (Jacoby et al., 2005), as well as semantic interference (Koutstaal \& Schacter, 1997; Norman \& Schacter, 1997). Some fMRI evidence suggests that older adults show reduced left VLPFC recruitment during relational memory encoding (Addis, Giovanello, Vu, \& Schacter, 2014) and reduced sensitivity to proactive interference in working memory (Jonides et al., 2000). Furthermore, several imaging studies investigating the effects of age on post-retrieval monitoring suggest that older adults underrecruit this process and show reduced DLPFC activity during episodic retrieval (Dulas \& Duarte, 2012; Fandakova, Lindenberger, \& Shing, 2013; Friedman, 2000). To our knowledge however, no previous studies have attempted to disambiguate the effects of age on separable executive control processes in associative memory under conditions of proactive interference. Thus, the extent to which age-related dysfunction in VLPFC and DLPFC operations contributes to older adults' susceptibility to proactive interference is unknown.

The present study investigated the effects of age on PFC-mediated executive control processes during associative memory retrieval under variable levels of proactive interference. 
During study, participants were shown objects paired with either a face or a scene. Each object was seen multiple times and the paired associate changed across viewings. There were two encoding conditions: High Interference and Low Interference. Interference level depended on how often an object was seen with the critical (most recent) associate vs. the lure. At test, participants were then shown each object again, as well as novel objects not seen previously. For each object, they were asked if it was old (seen previously) or new (not seen previously). They were then presented with a face and scene, and asked to determine which was most recently paired with the object.

We predicted the following:

1. Behaviorally, associative memory accuracy would be poorer for high interference compared to low interference across age groups, while item memory accuracy would not be affected by interference. Age-related associative memory accuracy deficits may be disproportionately evident for the high interference condition, in which demands on strategic retrieval processes are high.

2. We predicted that we would observe greater activity in the left mid-VLPFC for high interference than for low interference. However, we predicted this region would not distinguish between correct and incorrect associative memory judgments for high interference trials. This would be consistent with the suggestion that this region does not directly resolve proactive interference in service of associative memory performance.

3. We predicted that activity in the DLPFC would be greater for correct than incorrect associative memory trials under conditions of high interference. This would be 
consistent with the idea that this region supports post-retrieval monitoring processes or the number of post-retrieval decisions made.

4. While fMRI studies have demonstrated age-related alterations in VLPFC and DLPFC processes during associative memory tasks, no study has examined the differential effects of age on these processes. The present study was designed to disentangle the roles of age-related changes in these regions/processes in the detection and resolution of proactive interference in service of successful associative memory accuracy.

\section{Materials and Methods}

\subsection{Participants}

22 young adults, ages 19-35, as well as 22 older adults, ages 60-80, were recruited from local universities, science and health fairs, and community solicitation. Groups did not significantly differ in gender $\left[\chi^{2}(1, N=44)=0.86, p=0.35\right]$ or education $[t(42)=0.29, p=$ 0.77]. Group characteristics may be seen in Table 1. An additional young adult was excluded due to terminating the experiment early. An additional four older adults were excluded as well: one due to terminating the experiment early, one due to metallic hair dye that caused drop out in the MRI signal, one due to claustrophobia, and one due to excessive motion artifacts during scanning. All included participants were right-handed, native English speakers, with normal or corrected-to-normal vision (using MRI-compatible glasses when necessary). No participants reported a history of psychiatric or neurological disorders, uncontrolled vascular disease, psychoactive drug use, or use of CNS-active medication. Georgia Tech students were compensated with one credit per hour for their time. All other participants were compensated with $\$ 10$ per hour of their time, as well as an additional $\$ 5$ to cover travel expenses. All 
participants signed consent forms approved by the Georgia Institute of Technology Institutional Review Board.

\subsection{Neuropsychological Assessment}

All participants were administered a battery of standardized neuropsychological tests upon completion of the experiment. Tests specifically assessed memory ability and executive functioning to ensure no gross differences in performance due to cognitive impairment such as dementia in the older group. The battery included subtests from the Memory Assessment Scale battery (Williams, 1991): digit span forward and backward, list learning, recognition, recall and delayed recall, object recognition, recall, reproduction, and delayed recognition. Additionally,

the Trail making tests, A and B (Hedden \& Gabrieli, 2005), as well as the Controlled Oral Word Association Test ("FAS") (Benton, Hamsher, \& Sivan, 1983), were included. All participants were within the normal range for their age group.

\subsection{Materials}

Three hundred and sixty color photographs of nameable objects taken from Hemera Technologies Photo-Objects DVDs, or from the Internet via Google search, were used. All objects appeared on a white background. There was no overlap of multiple images depicting the same object. In addition, eight pictures of young adult faces ( 4 male, 4 female) and 8 pictures of scenes ( 4 indoor, 4 outdoor) were used as associates for the experiment. The faces were taken from the Max Planck Institute's FACES database (Ebner, Riediger, \& Lindenberger, 2010) and the scenes were taken from the SUN database (Xiao, Hays, Ehinger, Oliva, \& Torralba, 2010).

\subsection{Procedure}

The study was divided into two phases: Encoding and Retrieval. A practice session for each phase was administered before the beginning of the experiment to familiarize the 
participants with the tasks and to ensure they could sufficiently perform the memory task before entering the scanner. Only the Retrieval phase of the study was scanned. Stimuli were counterbalanced across participants, such that each object appeared in different conditions across participants. There were 240 objects studied during Encoding, as well as an additional 120 new objects seen at Retrieval.

The Encoding phase was divided into 5 blocks, which were administered outside of the scanner. Each block consisted of 240 trials, displaying all of the to-be-studied objects. For each trial, participants were shown an object on the left and either a face or a scene on the right for $3000 \mathrm{~ms}$ (Figure 1). Participants were asked to imagine the images together and to rate how easy or hard it was to do this. For example, when participants were shown an object with a face, they were instructed to imagine that person interacting with that object. Likewise, when they were shown an object with a scene, they were instructed to imagine that object in that location. This incidental orienting task was meant to facilitate encoding of both the images in the pair and to reduce age-related differences in self-initiated processing at encoding (Naveh-Benjamin, Brav, \& Levy, 2007). There were three options: 1 = Easy, 2 = Medium, 3 = Hard. Each trial was followed by a fixation cross for $500 \mathrm{~ms}$. Participants placed their right index finger on 1 , their right middle finger on 2, and their right ring finger on 3. Objects were presented in a random order within each block. As each block was about 14 minutes, we also provided a 30 second break halfway through each block to prevent fatigue. The total time for the Encoding phase was about an hour and 15 minutes.

During Encoding, objects were divided evenly across the High and Low Interference conditions (120 objects per condition). For the Low Interference condition, each object was paired four times with one associate (e.g. a face) and once with an associate of the opposite type 
(e.g. a scene). Critically, the final time the object was viewed, it was shown with the most frequently paired associate (in this example, a face). For example, a picture of a banana may be paired first with a female face, then with a picture of an office, and then with the female face for the last 3 blocks. Thus, during Retrieval, there may be some interference between the female face and the office, but the more highly associated image would also be the critical (i.e. most recent) associate. For the High Interference condition, each object was paired three times with one associate (e.g. a scene) for the first three blocks, and then paired with the other associate (e.g. a face) in the final two blocks ${ }^{1}$. For example, a wagon may be paired with a forest for the first three blocks, but paired with a male face for the final two blocks. Thus, at Retrieval, there would be a strong association between the object and the irrelevant image (forest), creating a high level of interference.

After the Encoding phase, participants entered the scanner to start the Retrieval phase. The structural scan (see 2.6 fMRI Analysis) was taken after the Retrieval phase to minimize delay between Encoding and Retrieval. Retrieval was divided into four blocks, consisting of 90 trials each (60 old items, 30 new items), for a total of 360 trials (Figure 2). The inclusion of new items allowed us to look for age-related old-new effects that may inform our data, as well as item recognition accuracy. Further, activity for correct rejections ("new" responses to unstudied items) may serve as a baseline condition relative to the other conditions of interest (as done in Duarte, Graham, \& Henson, 2010; Duarte, Henson, \& Graham, 2008). That is, there should be little to no evidence of processing such as post-retrieval selection or monitoring during a correct rejection trial. On each trial, participants were shown an object in the center of the screen, which remained there across the entire trial, until the final fixation cross. Participants were first asked to

\footnotetext{
${ }^{1}$ This design was chosen after extensive piloting showed that this manipulation produced above chance level performance for both age groups.
} 
determine if the object was Old (button 1) or New (button 2). This prompt was on the screen for $2000 \mathrm{~ms}$. Then the question, "Which image was paired with this object most recently?" appeared for $1000 \mathrm{~ms}$. Participants were instructed that they could still respond to the old-new question during this time. Then a face and a scene appeared below the object for $3000 \mathrm{~ms}$. The image on the left corresponded to button 1 and the image on the right corresponded to button 2. For half the participants, the face was shown on the left and the scene on the right; for the other half this was reversed. The two images were always the critical stimulus and the lure for old objects. Participants were still shown this question even if they said the object was new. For novel objects, participants were instructed to simply look at images and press either button. At the end of the trial, the object was removed and there was a $500 \mathrm{~ms}$ fixation cross, indicating the next trial was about to begin. Objects were presented in pseudorandom order within each block so that no more than 5 trials of the same condition (i.e. high interference, low interference, new item) were presented sequentially. Each block lasted about 10 minutes, for a total of 40 minutes.

For all behavioral analyses, significant interactions at an alpha $(\alpha)$ level of 0.05 were followed up with subsidiary ANOVAs and t-tests to determine the source of the effects. Where appropriate, reported p-values were corrected using Huynh-Feldt corrections.

\section{5 fMRI Acquisition}

Scanning was performed on a 3-T Siemens TIM Trio system. Functional data were acquired using a gradient echo pulse sequence (37 transverse slices oriented along the anterior-posterior commissural axis with a 30 degree upward tilt to avoid the eyes, repetition time of $2 \mathrm{~s}$, echo time of $30 \mathrm{~ms}, 3$ × 3 x $3.5 \mathrm{~mm}$ voxels, $0.8-\mathrm{mm}$ interslice gap). Four Retrieval blocks of 303 volumes were acquired. The first 5 volumes of each block were discarded to allow for 
equilibration effects. A high-resolution T1-weighted magnetization-prepared rapid acquisition gradient echo (MPRAGE) image was collected for normalization (see 2.6 fMRI Analysis).

\section{6 fMRI Analysis}

Data were analyzed with SPM8 (http://www.fil.ion.ucl.ac.uk/spm/software/spm8/). Images were corrected for differences in slice timing acquisition using the middle slice of each volume as the reference, spatially realigned, and resliced with respect to the first volume of the first block. Each participant's MPRAGE scan was coregistered to the mean echo planar imaging (EPI), produced from spatial realignment. Each coregistered structural scan was then segmented using the Diffeomorphic Anatomical Registration Through Exponentiated Lie algebra (DARTEL) SPM 8 toolbox (Ashburner, 2007). DARTEL is a suite of tools fully integrated with SPM 8, which the SPM 8 manual recommends over optimized normalization, to achieve sharper nonlinear registration, for intersubject alignment. This method also achieves better localization of fMRI activations in Montreal Neurological Institute [MNI] space. This method has been used successfully in several previous studies with various healthy and neurological populations (Pereira et al.; Yassa \& Stark, 2009). Briefly, the gray and white matter segmented images were used to create a study-specific template using the DARTEL toolbox and the flow fields containing the deformation parameters to this template for each subject were used to normalize each participant's realigned and resliced EPIs to MNI space. Normalized EPI images were written to $2 \times 2 \times 2 \mathrm{~mm}$ and smoothed with an $8 \mathrm{~mm}$ full-width at half-maximum isotropic Gaussian kernel. The EPI data were then high-pass filtered to a minimum of $1 / 128 \mathrm{~Hz}$ and grand mean scaled to 100 .

Statistical analysis was performed in 2 stages. While there were two response prompts, activity was only modeled to the onset of the first prompt (old-new), as participants were aware 
of the second prompt and may have been anticipating their associative memory responses. In order to capture both the old-new effects, as well as the associative memory effects, neural activity was modeled as a series of 4-s epochs for the various event types and convolved with a canonical hemodynamic response function ${ }^{2}$. We have previously demonstrated (Dulas \& Duarte, 2014) that this method shows both old-new (i.e. item memory) and associative memory effects that are consistent with those reported in studies that have analyzed these effects separately using the separate trial approach (Dobbins, Foley, Schacter, \& Wagner, 2002; Hayes, Buchler, Stokes, Kragel, \& Cabeza, 2011). We reasoned that the duration of the peak BOLD response for postretrieval effects supporting associative memory would be sustained relative to, for example, item recognition effects. Importantly, different contrasts were used to separate item recognition from associative memory. Consistent with previous studies (Dulas \& Duarte, 2012, 2014; Morcom, Li, \& Rugg, 2007) the contrast between item hits and correctly rejected new items was used to index item recognition. By comparison, the contrast between associative correct and associative incorrect trials was used to index associative memory accuracy. Thus, we capture both old-new effects and associative memory effects during the same epoch via distinct contrasts.

The time courses were then down-sampled to the middle slice to form the covariates for the general linear model. For each participant and block, 6 covariates representing residual movement-related artifacts, determined by the spatial realignment step, were included in the first-level model to capture residual (linear) movement artifacts. Voxel-wise parameter estimates for these covariates were obtained by restricted maximum-likelihood estimation, using a

\footnotetext{
${ }^{2}$ In a separate analysis of these data not shown here, we analyzed the old-new response prompt and the associative memory response prompt with separate onsets using delta (stick) functions. The old-new regressor analysis showed robust old-new effects but failed to show the PFC effects sensitive to associative interference and/or accuracy, suggesting these effects onset later, after item recognition. Analyses for the associative memory regressor were quite similar to our reported 4-s box car approach, showing both interference and associative memory effects. Indeed, correlations between the betas observed for our original boxcar analysis and the late regressor showed these two analyses were extremely similar for each contrast [ $\left.r^{\prime} s>0.96, p^{\prime} s<0.001\right]$.
} 
temporal high-pass filter (cutoff $128 \mathrm{~s}$ ) to remove low-frequency drifts and modeling temporal autocorrelation across scans with an $\mathrm{AR}(1)$ process.

Contrasts of the parameter estimates for each participant were submitted to the second stage of analysis (treating participants as a random effect). A mixed-ANOVA model was created for the test period that allowed us to examine both within-group effects and group interactions. The 4 x 2 model included factors of Condition (High Interference Associative Correct, High Interference Associative Incorrect, Low Interference Associative Correct, and Correct Rejections) and Group (young, old). This ANOVA model allowed us to not only assess interference effects (i.e. High Interference Associative Correct > Low Interference Associative Correct > Correct Rejections) using directional t-test comparisons, but also determine whether regions sensitive to interference were also sensitive to accuracy (i.e. High Interference Associative Correct > High Interference Associative Incorrect). Importantly, this approach also allows parameter estimates for all conditions to be plotted for the same region, as shown in several figures. There were insufficient numbers of "New" responses to studied (misses), "Old" responses to unstudied (false alarms) objects, and Associative Incorrect responses to Low Interference trials for all participants to examine separately; thus these were also not included in the ANOVA. It should also be noted that there were also insufficient numbers of trials for the above categories to analyze face and scene associations separately, thus these were collapsed. Given that we had no a priori predictions about associative memory encoding relative to baseline, we purposefully did not include a "baseline" condition (e.g. fixation trials). Importantly, the inclusion of null event/fixation trials to measure inter-stimulus "baseline" is not necessary in fMRI designs if the contrast of interest is between task conditions (Henson, 2007). 
Covariates modeling the mean across conditions for each participant were also added to each model, to remove between-subject variance of no interest. A weighted least squares estimation procedure was used to correct for inhomogeneity of covariance across within-group conditions and inhomogeneity of variance across groups.

The SPMs for main effect effects across groups were masked exclusively with the SPMs for all relevant interactions, using a liberal uncorrected threshold of $P<0.05$ for the masks in order to restrict memory effects to those "common" (i.e. similar size) across conditions and groups. This ensures that supposed "common" effects do not in fact represent regions in which groups or conditions actually interact. To correct for multiple comparisons, all results were thresholded at $p<0.001$ with a cluster extent of 17 , which yielded whole-brain results corrected for multiple comparisons at $\mathrm{p}<0.05$. We derived this threshold via Monte Carlo simulations to correct for Type I and Type II errors (Slotnick, Moo, Segal, \& Hart, 2003)³.

Further, all effects were confirmed via inclusive masking $(\mathrm{p}<0.01)$ with each side of the effect (i.e. young and older adult effects) in order to elucidate the source of interactions in a given contrast (e.g. Young > Old: High > Low Interference), allowing us to determine whether an effect was driven by a group crossover, or whether an effect was in fact larger in one group than another, as well as to ensure that main effects across groups were reliable for each group.

Maxima of significant clusters were localized on individual normalized structural images. Neural activity from these maxima was plotted for Associative Correct, Associative Incorrect, and Correct Rejection responses. Neural activity reflected the parameter estimates for the convolved regressors and had arbitrary units.

\section{RESULTS}

\footnotetext{
${ }^{3}$ Our XYZ matrix dimensions were $68 \times 68 \times 37$, with a $3 \times 3 \times 3.5 \mathrm{~mm}$ voxel size resampled to $2 \times 2 \times 2 \mathrm{~mm}$. The Gaussian full-width half-maximum was set to 12 , which was the most conservative (highest) value computed using the t-statistic maps associated with the contrasts of interest. 1000 simulations were run.
} 


\subsection{Neuropsychological Assessment Results}

Group characteristics and results for neuropsychological tests are shown in Table 1. All participants scored within one standard deviation of age-adjusted normative averages for all neuropsychological tests. Older adults exhibited significantly poorer performance than young adults on several tests including Trails A \& B, Visual Recognition, Delayed Visual Recognition, and Visual Reproduction [t(42)'s $>2.38$, p's $<0.03$ ]. There were no other significant group differences [t(42)'s $<1.00, p$ 's $>0.32]$.

\subsection{Behavioral Results}

Mean proportions for correct and incorrect associative memory judgments, incorrect "new" judgments to studied items (misses), and correct "new" judgments to novel items (correct rejections) are shown in Table 2. Item recognition accuracy was estimated by the Pr measure of discriminability (Snodgrass \& Corwin, 1988), i.e. p(hits) - p(false alarms) for High and Low interference conditions, where "hits" include both associative correct and associative incorrect responses. Associative memory accuracy was estimated using the proportion of hits associated with correct associative memory decisions, excluding misses (i.e. incorrect "new" judgments to old items), where 0.5 is the level of chance (e.g. Morcom et al., 2007; Swick, Senkfor, \& Van Petten, 2006).

An ANOVA of item recognition accuracy, with factors of Interference (High, Low) and Age (Young, Old) revealed a marginal effect of Age $[F(1,42)=3.63, p=0.06]$, but no other significant effects [ $F$ 's $<2.24, p$ 's $>0.14]$. Thus, interference had no effect on item memory. Further older adults only showed modest declines in item recognition accuracy.

A similar ANOVA for associative memory accuracy revealed a significant main effect of Interference $[F(1,42)=225.72, p<0.001]$ and a marginally significant effect of Age $[F(1,42)=$ 
3.90, $p=0.06]$. However, the interaction between Age and Interference was not reliable $[F(1,42)$ $=0.36, p=0.55]$. Thus, while older adults did perform more poorly overall, and interference did greatly affect associative memory accuracy, older adults were not significantly more impaired for the High, compared to Low, Interference condition.

We also conducted analyses of response times (RTs) to the associative memory prompt. The response times for correct and incorrect associative memory responses to High and Low interference trials are shown in Table 2. We conducted an ANOVA with factors of Interference (High, Low), Associative Memory Accuracy (Correct, Incorrect), and Age (young, old). Results showed main effects of Interference $[F(1,42)=5.55, p=0.02]$, Accuracy $[F(1,42)=71.59, p<$ 0.001], and Age $[F(1,42)=7.60, p=0.01]$, as well as an interaction between Interference and Accuracy $[\mathrm{F}(1,42)=44.01, \mathrm{p}<0.001]$. All other effects were not reliable $[F$ 's $<1.49, p$ 's $>$ 0.23]. Follow-up analyses across groups revealed that Correct trials were faster than Incorrect trials for both High and Low Interference conditions [t(43)'s $>3.85, p$ 's $<0.001]$. However, for Correct trials, High Interference trials were slower than Low Interference trials $[t(43)=6.68, p<$ 0.001], but the reverse was true for Incorrect trials, where High Interference trials were faster than Low Interference trials $[t(43)=5.05, p<0.001]$. It should be noted that RTs to the associative memory prompt were positively correlated with RTs to the old-new response for all trial types $[r$ 's $>0.46, p$ 's $<0.002]$, suggesting these two sets of responses are not independent.3.3 fMRI Results

In line with previous studies (Dulas \& Duarte, 2012, 2014; Duverne, Habibi, \& Rugg, 2008; Morcom et al., 2007), we first compared Associative Correct (ACs) responses and Correct Rejections (CRs). However, while contrasts comparing AC and CR responses are robust, they may represent both associative and item memory effects. Thus, in addition, we examined 
associative memory specific effects (Associative Correct vs. Associative Incorrect responses) as we have done previously (Dulas \& Duarte, 2012, 2014).

\subsubsection{Old-New Effects Across Interference}

The contrast of $\mathrm{AC}>\mathrm{CR}$ across conditions and groups showed the typical pattern of recognition memory activity in bilateral PFC, lateral and medial parietal cortex. These data are not presented.

Young adults showed greater old-new effects than older adults in the right middle frontal gyrus, right orbitofrontal gyrus, and right parietal cortex. Older adults showed greater activity than the young in the bilateral aspects of the middle frontal gyrus (posterior relative to the middle frontal region showing greater activation in the young), left superior parietal cortex, as well as bilateral middle occipital cortex. These regions are shown in Table 3.

\section{$\underline{\text { 3.3.2 Interference Effects }}$}

Several regions showed greater effects for High Interference compared to Low Interference trials (Table 4). These regions included, most notably, the left parahippocampal cortex, the left superior parietal cortex, and the left mid-VLPFC (Figure 3). No regions showed evidence of greater effects for Low Interference compared to High Interference trials across groups. No regions showed significant interactions between age and interference level.

\section{$\underline{\text { 3.3.3 Effects of Interference on Associative Memory Accuracy }}$}

Associative accuracy effects (AC > AI) were only analyzed for High Interference trials, as most participants did not have enough Low Interference Incorrect trials to analyze. These results are shown in Table 5. Across groups, Associative Correct trials showed greater activity than Associative Incorrect trials in several regions, most notably the left hippocampus (Figure 4). 
There were many regions showing greater associative memory accuracy effects for the young than the old, most notably left DLPFC (BA 9) and the anterior PFC (BA 10) (Figure 5). No regions showed greater associative memory accuracy effects for the old than young.

The left mid-VLPFC was sensitive to interference but not to associative memory accuracy (Figure 6). Given our particular interest in determining whether the left mid-VLPFC also supported associative memory accuracy under conditions of high interference, we reanalyzed these contrasts at a reduced threshold $(\mathrm{p}<0.005)$. However, even at a reduced threshold, there was no evidence of accuracy effects in the left VLPFC. Interestingly, while there was no difference between High Interference Associative Correct and Incorrect trials in this region, both were significantly greater than Low Interference Associative Correct trials, as revealed by inclusive masking $(\mathrm{p}<0.01)$. It should be noted that, given that our accuracy and interference contrasts both included high interference correct trials, these contrasts are not fully independent. However, the contrasts demonstrated striking dissociations in their patterns of activity as well as a dissociation with respect to age-group differences.

Interestingly, correlational analyses showed a marginal correlation between the hippocampal effects observed across groups and high interference associative memory accuracy in the young $[r=0.37, p=0.09]$, but not the old $[r=-0.24, p=0.27]$. That said, none of the PFC regions showing accuracy or interference effects were correlated with associative memory accuracy, or behavioral interference effects ( $r$ 's $<0.23, p$ 's $>0.13)$. However, note that a null correlation between brain and behavior is a weak platform from which to draw conclusions about regions not contributing to behavior. It should be noted that our failure to find additional correlations between our imaging and behavioral results may be due to relatively low variance in associative memory performance, particularly in older adults. 


\section{DISCUSSION}

The present study investigated the effects of aging on overcoming proactive interference in associative memory. We were particularly interested in assessing the effects of aging on different PFC operations thought to be involved in detecting and overcoming proactive interference. As predicted, associative memory accuracy was poorer for high compared to low interference trials across groups. However, age-related memory deficits were relatively small (only marginally significant), and there was no interaction between age and interference. Imaging result showed that, across groups, increasing levels of interference (High > Low > CR) resulted in increased recruitment of the left mid-VLPFC. However, the left mid-VLPFC did not dissociate correct from incorrect associative memory responses. Older adults showed reductions in associative memory accuracy effects in the left DLPFC and anterior PFC. These results and their implications are discussed below.

\subsection{Behavioral Results}

As has been demonstrated previously (Jacoby et al., 2005; Jacoby, Wahlheim, Rhodes, Daniels, \& Rogers, 2010; Nee et al., 2007; Yonelinas \& Jacoby, 2012), associative memory accuracy was poorer for high levels of proactive interference compared to low. This was true for both young and older adults. Thus, when interference from a to-be-ignored association is high, the ability to correctly remember, or select, the weaker but goal-relevant association is reduced. Interestingly, there was only a marginal main effect of age across both item and associative memory, with no interaction between age and interference level.

The finding that age-related associative memory deficits in the present study were relatively small stands in contrast to previous work from our lab (Duarte et al., 2008; Dulas \& Duarte, 2011, 2012, 2013, 2014; Dulas, Newsome, \& Duarte, 2011), as well as others (e.g. 
Chalfonte \& Johnson, 1996; Glisky, Rubin, \& Davidson, 2001; see Mitchell \& Johnson, 2009 for review; Naveh-Benjamin, 2000; Naveh-Benjamin et al., 2007) that suggests associative memory is disproportionately impaired with age. The lack of an interaction between age and interference level also stands in contrast to previous work suggesting older adults show greater susceptibility to mnemonic interference (Bowles \& Salthouse, 2003; Jacoby et al., 2005; Jonides et al., 2000; Lustig \& Jantz, 2015; Lustig et al., 2001; May et al., 1999). One possible reason for these discrepant findings could be that we provided participants with an integrative encoding task, wherein they had to imagine the images interacting, and rate how easy or hard it was to do so. Previous evidence has suggested that providing effective encoding strategies may reduce agerelated associative memory deficits, given that older adults may simply fail to self-initiate such strategies (Glisky \& Kong, 2008; Glisky et al., 2001; Hay \& Jacoby, 1999; Naveh-Benjamin et al., 2007). That said, marginal age-related differences did persist, falling in line with evidence that environmental support at encoding is insufficient to fully overcome age-related associative memory deficits (Dulas \& Duarte, 2013, 2014; Naveh-Benjamin et al., 2007). Previous behavioral evidence has shown that age-related associative memory deficits are only fully ameliorated when support is provided at both encoding and retrieval (Naveh-Benjamin et al., 2007). Future work, in which both encoding and retrieval are supported, may be able to determine whether age-related declines in overcoming proactive interference are due to binding deficits (Naveh-Benjamin, 2000; Old \& Naveh-Benjamin, 2008), or simply a failure to engage in effective retrieval strategies.

Lastly, response time data revealed an interesting interaction between accuracy and interference. While older adults were slower overall, both groups showed faster correct responses to low, compared to high interference conditions, but the reverse for incorrect responses (i.e. 
high faster than low). This is in line with studies of proactive interference in working memory, which have shown slower correct response times for high compared to low interference trials (see Jonides \& Nee, 2006 for review). As suggested previously, strategic retrieval processes, like post-retrieval monitoring, are engaged when participants are close to their decision criterion. When interference is low, participants are likely closer to their decision criterion for incorrect than correct trials, in line with previous associative memory studies (Achim \& Lepage, 2005;

Dulas \& Duarte, 2012, 2014; Henson, Rugg, Shallice, Josephs, \& Dolan, 1999). However, under conditions of high interference, correct trials likely reflect instances in which participants retrieve both associates, and must engage strategic operations to overcome the interfering lure. These results are also in line with our accuracy data, which similarly failed to show an interaction between interference and age, suggesting older adults were not disproportionately affected by interference in this study.

\subsection{Imaging Results}

4.2.1 Executive control contributions to interference resolution: similarities across age

Both young and older adults exhibited a network of PFC regions that showed greater activity with increasing proactive inference, including the anterior cingulate, left dorsal premotor cortex, and, most notably, the left mid-ventrolateral prefrontal cortex. Previous research has suggested that the left mid-VLPFC, extending into the inferior frontal sulcus (Barredo, Oztekin, \& Badre, 2013), may be involved in post-retrieval selection (Badre et al., 2005; Badre \& Wagner, 2007; Barredo et al., 2013; Dobbins, Rice, Wagner, \& Schacter, 2003). This process is said to be engaged when multiple competing representations are simultaneously active in memory and a goal-appropriate representation must be chosen, such as in a proactive interference task (Badre \& Wagner, 2007). This work has differentiated the selection process 
attributed to the left mid-VLPFC (BA 44/45) from the anterior VLPFC (BA 47), which is thought to be involved in controlled retrieval processes engaged when association strength is low (Badre et al., 2005; Badre \& Wagner, 2007). We contend that the left VLPFC region implicated in the present study, which similarly extends into the inferior frontal sulcus, is more spatially aligned with the mid-VLPFC area associated with post-retrieval selection than the anterior VLPFC. Indeed, the peak voxel of the left mid-VLPFC reported in the present study $[-50,24,31]$ is extremely close to the peak mid-VLPFC voxel previously reported to respond to selection demands across multiple tasks $[-51,15,33]$, which was also seen in BA 44/45, extending into the inferior frontal sulcus (Badre et al., 2005; Barredo et al., 2013) ${ }^{4}$. It should be noted that this region shows activity across many tasks requiring resolution of interference (Atkins \& ReuterLorenz, 2011; Blumenfeld \& Ranganath, 2007; Jonides \& Nee, 2006; Thompson-Schill, Bedny, \& Goldberg, 2005) and is likely not memory-specific (Badre et al., 2005; Badre \& Wagner, 2007). While it is the case that target association strength likely differs between high and low interference trials, given that target associations were paired twice as often for low than high interference trials, the VLPFC area tracking interference is more caudal than that implicated in controlled retrieval (Badre \& D'Esposito, 2007; Badre et al., 2005; Badre \& Wagner, 2007; Barredo et al., 2013). Indeed the anterior VLPFC was not implicated in retrieval in the current study.

While the left mid-VLPFC has been repeatedly implicated in the successful resolution of proactive interference (see Badre \& Wagner, 2007 for review), few studies have investigated whether this process contributes directly to successful memory retrieval. In the present study, the

\footnotetext{
${ }^{4}$ To test if the left mid-VLPFC region previously shown to be sensitive to interference (Badre et al., 2005; Barredo et al., 2013) was indeed proximal to our own, we conducted a Region of Interest (ROI) analysis using this peak voxel as a seed. With a sphere of $8 \mathrm{~mm}$, results showed significant High $>$ Low Interference effects (FWE $<0.05)$ in this left mid-VLPFC ROI, but no associative memory accuracy effects.
} 
left mid-VLPFC showed no difference between correct versus incorrect associative memory responses for high interference trials, and both correct and incorrect responses showed significantly greater activity than low interference associative correct responses. This finding is in line with previous studies from the working memory domain (Atkins \& Reuter-Lorenz, 2011; Oztekin et al., 2009), which suggest this region simply acts to index the level of interference, but does not contribute directly to resolution in service of accurate memory retrieval. It should be noted, however, that both lesion (Thompson-Schill et al., 2002) and TMS (Feredoes et al., 2011) studies have shown that disruption to the left VLPFC results in increased susceptibility to interference. Thus, it is likely that the role that the mid-VLPFC plays in indexing interference is ultimately necessary for retrieval success. However, its role may be less direct than that of some other PFC regions that contribute to resolution of competition between retrieved memory representations in support of associative memory accuracy. In the present study, the left midVLPFC showed a similar sensitivity to interference as the anterior cingulate, a region thought to be involved in indexing the amount of interference/conflict present across multiple tasks/domains (Bunge, Burrows, \& Wagner, 2004; Kim, Johnson, \& Gold, 2012; Ochsner, Hughes, Robertson, Cooper, \& Gabrieli, 2009; van Veen \& Carter, 2002). Thus, these regions may serve similar roles in indexing interference, though potentially across different domains (Nelson, Reuter-Lorenz, Sylvester, Jonides, \& Smith, 2003). That said, our contrasts used to define interference and accuracy effects were not fully independent; however, these contrasts showed a qualitative dissociation between regions, as well as differences with respect to age group differences. Still, a definitive dissociation between regions showing interference and accuracy sensitivity requires further investigation.

\subsubsection{Executive control contributions to interference resolution: differences across age}


Compared to the young, older adults showed reduced associative memory accuracy effects under conditions of high interference in several regions, including the precentral gyrus, the DLPFC, and the anterior PFC. This was true despite only marginal impairments in associative memory accuracy. However, the age-related under-recruitment of the DLPFC is consistent with previous results from a temporal order retrieval study, potentially suggesting that dysfunction in this region may underlie many kinds of associative memory impairments in the old (Rajah, Languay, \& Valiquette, 2010). Several studies have suggested that the DLPFC and anterior PFC support post-retrieval monitoring processes (Achim \& Lepage, 2005; Atkins \& Reuter-Lorenz, 2011; Fandakova et al., 2013; Fletcher \& Henson, 2001; Henson et al., 2000; Henson, Shallice, et al., 1999; Turner et al., 2008). Post-retrieval monitoring involves the maintenance, manipulation, and evaluation of retrieved memory representations, particularly when a participant is at or near his/her decision criterion (Henson et al., 2000). In the present study, participants were likely closer to their decision criterion on correct trials for the High Interference condition, as incorrect associative memory responses may reflect trials wherein the lure was so salient that it was immediately selected without additional processing of temporal context. These findings also fit with a decision-making account of DLPFC activity, which suggests the DLPFC is sensitive to the number of internal decisions that need to be made (Dobbins \& Han, 2006; Han et al., 2009). On correct trials, young adults may engage in the evaluation of both associates in order to successfully select the goal-relevant one, determining if they were both previously encountered, which one was encountered more recently, and how confident they are in their decision. Interestingly, emerging evidence has suggested a possible hierarchical organization to the prefrontal cortex (Badre \& D'Esposito, 2009; Christoff, Keramatian, Gordon, Smith, \& Madler, 2009; Domenech \& Koechlin, 2015), wherein more 
dorsal and anterior subregions, particularly left lateralized (Bunge, Helskog, \& Wendelken, 2009), of the PFC are recruited as demands on relational integration increase (i.e. relations of relations vs. item-item relations). Although we did not tax demands on relational integration in our task directly, the successful resolution of interference may have required processing of multiple levels of associations. Regardless, older adults may be less likely to engage in these additional executive processes. That said, despite the under-recruitment of the DLPFC and anterior PFC, older adults showed only marginal associative memory declines, which were not affected by interference. Thus, while these PFC regions are likely involved in resolving interference, as evidenced by the pattern of activity in the young, the lack of a major group difference in performance suggests these regions are not the sole, or perhaps primary, contributor to successful interference resolution. Future work TMS and/or lesion studies may be better able to determine the necessity of these PFC regions in associative memory accuracy and overcoming proactive interference.

While not an initial focus of this study, previous studies have shown increased activity in the precentral gyrus related to response conflict/interference, as opposed to conceptual interference (see Badre \& D'Esposito, 2007 for review; Hazeltine, Bunge, Scanlon, \& Gabrieli, 2003; Ullsperger \& von Cramon, 2001). It is possible that increased conflict at a conceptual level, i.e. proactive interference, also results in increased response conflict. However, future work investigating age-related changes on the differential roles of the precentral and lateral prefrontal regions in overcoming various types of interference, i.e. response vs. conceptual level, may further address these questions.

Interestingly, we found no regions in which older adults showed greater associative memory or interference effects compared to the young. Some previous evidence has suggested 
older adults may show increased activity, particularly in the PFC, compared to young adults and that this over-recruitment supports performance (Cabeza, Anderson, Locantore, \& McIntosh, 2002; Davis, Dennis, Daselaar, Fleck, \& Cabeza, 2008; Grady, McIntosh, \& Craik, 2005; McDonough, Wong, \& Gallo, 2012; Rajah \& D'Esposito, 2005). However, there is emerging evidence suggesting that when large group differences in performance are controlled or minimal, there is little evidence of over-recruitment of PFC activity related to associative memory accuracy (Cansino, Hernandez-Ramos, \& Trejo-Morales, 2012; Dulas \& Duarte, 2012). Given that the age differences in associative memory performance in the present study were relatively small, our results add to the assertion that persevered memory performance in aging does not necessitate prefrontal compensation (Duverne, Motamedinia, \& Rugg, 2009; Duzel, Schutze, Yonelinas, \& Heinze, 2011). It should be noted that older adults showed increased old-new effects compared to young adults across interference levels in several regions. However, none of these regions showed significant correlations with memory performance, although this is a null finding. Nonetheless, the over-recruitment observed in older adults, particularly given that it was found in regions adjacent to those showing old-new effects across groups, is most consistent with an age-related decrease in neural efficiency (Grady, 2012; Logan, Sanders, Snyder, Morris, \& Buckner, 2002; Morcom et al., 2007; Rypma, Eldreth, \& Rebbechi, 2007).

Taken together, our results point toward the need for a more nuanced perspective of the neurocognitive model of aging than the frontal aging hypothesis (Raz et al., 1997; West, 1996). The present study adds to growing evidence (Dulas \& Duarte, 2013, 2014; Leshikar, Gutchess, Hebrank, Sutton, \& Park, 2010; Li, Morcom, \& Rugg, 2004; MacPherson, Phillips, \& Della Sala, 2002; Rugg \& Morcom, 2005) that older adults can recruit at least some PFC processes, particularly those mediated by the VLPFC (Dulas \& Duarte, 2014; Leshikar et al., 2010; Logan 
et al., 2002; Rypma \& D'Esposito, 2000), to a similar extent as the young, even in the presence of dysfunction in DLPFC (Rypma \& D'Esposito, 2000) and anterior PFC (Dulas \& Duarte, 2014; Fandakova et al., 2013).

The finding that older adults showed similar recruitment of the left VLPFC as the young stands in contrast to previous evidence that older adults show dysfunction in this region (Addis et al., 2014; Jonides et al., 2000). For example, recent evidence from item-relatedness research has shown similar results to our own in young adults, wherein the left VLPFC showed greater activity for related vs. unrelated item lures, but did not distinguish correct from incorrect responses to related lures, while older adults, who performed significantly more poorly on the task, did show accuracy effects (correct > incorrect to related lures) in this left VLPFC region (Bowman \& Dennis, 2015). However, in the present study, age-related associative memory accuracy differences were relatively small, suggesting differences in VLPFC recruitment may in fact reflect differences in performance, rather than the effects of healthy aging (Rugg \& Morcom, 2005). As mentioned previously (section 4.1), behavioral evidence suggests that older adults may be less likely to self-initiate effective strategic control operations (Luo \& Craik, 2008; NavehBenjamin et al., 2007). Previous evidence, however, suggests that providing environmental support, similar to our encoding task, may attenuate age-related PFC under-recruitment (Logan et al., 2002). Further, the present finding falls in line with previous evidence from our own lab demonstrating that young and older adults showed similar VLPFC effects at retrieval following encoding support (Dulas \& Duarte, 2014). What is unclear, however, is whether the age-related alterations in DLPFC and anterior PFC functioning are intractable, or simply represent a failure by older adults to spontaneously engage in the "high-order" processes supported by these regions (Badre \& D'Esposito, 2007, 2009). Accumulating evidence shows that only by providing 
environmental support at both encoding and retrieval do older adults perform similarly to the young (Naveh-Benjamin et al., 2007). Thus, it is possible that manipulations encouraging highorder processing during the retrieval phase may cue older adults to initiate these processes in a similar manner to the young. However, it is also possible that the dorsolateral and anterior PFCs are simply more susceptible to age-related dysfunction than the VLPFC. Future work investigating the role of retrieval support in overcoming proactive interference may be better able to address whether these changes are truly intractable, or simply reflect a failure to initiate more complex retrieval strategies.

\subsubsection{Contributions of core episodic memory regions to interference resolution: no age} $\underline{\text { differences }}$

The left superior parietal cortex and the parahippocampal cortex showed increased activity with higher levels of interference, while the hippocampus was sensitive to associative memory accuracy and showed a marginal correlation with associative memory performance in young adults. The superior parietal cortex has been suggested to index top-down attention to memory (Cabeza, 2008; Ciaramelli, Grady, \& Moscovitch, 2008; Vilberg \& Rugg, 2008), often in situations when participants have more difficult memory decisions (Burianova, Ciaramelli, Grady, \& Moscovitch, 2012; Ciaramelli et al., 2008). These results may suggest that, under conditions of high interference, participants may have more difficulty determining the correct associate and therefore must apply more attention toward the products of retrieval in order to resolve the presence of conflicting information. However, given that this region was not sensitive to associative memory accuracy, the present results suggest top-down attention alone is insufficient to mitigate this competition and successfully resolve interference. 
It has been well established that the hippocampus is involved in successful associative memory retrieval (Diana, Yonelinas, \& Ranganath, 2007, 2009; Eichenbaum, Yonelinas, \& Ranganath, 2007; Staresina, Henson, Kriegeskorte, \& Alink, 2012; Yonelinas, Otten, Shaw, \& Rugg, 2005). Previous evidence also suggests that the medial temporal lobe may also be critical for overcoming interference in memory (Fandakova et al., 2013; Oztekin et al., 2009). Our finding that the hippocampus dissociated correct from incorrect associative memory responses under conditions of high interference suggests that the successful resolution of interference requires retrieval of tightly bound memory representations. That is, participants likely need to retrieve multiple pieces of contextual information, for example both whether associates were paired with the object, as well when they were seen, to correctly determine which associate was the most recent under conditions of high interference. The lack of group differences in this region stands in contrast to previous evidence showing age-related dysfunction in the hippocampus, be it under-recruitment (Daselaar, Fleck, Dobbins, Madden, \& Cabeza, 2006; Dennis, Kim, \& Cabeza, 2008; Dulas \& Duarte, 2014; Grady et al., 2005) or over-recruitment (Cabeza et al., 2002). Given that older adults showed only a marginal decline is associative memory performance in our study, these results support the idea that age-related differences in hippocampal function may simply be a product of performance, rather than an effect of healthy aging, per se (see Rugg \& Morcom, 2005 for review).

A remaining question that the present study is unable to address is whether age-related declines in overcoming interference are related, at least in part, to differences in encoding. Previous evidence from young adults using a similar encoding task showed increased activity in left VLPFC across encoding repetitions of previously seen stimuli, possibility reflecting interference processing during study (Zeithamova \& Preston, 2010). Studies looking at both 
encoding and retrieval have also shown that VLPFC activity during encoding may reduce perceptual reactivation of distractors during retrieval (Kuhl, Bainbridge, \& Chun, 2012). It is important to note that neural correlates of associative memory encoding depend on the manner in which memory is assessed during retrieval and retrieval activity depends on how associations are encoded (see Rugg, Johnson, Park, \& Uncapher, 2008 for review). Thus, it is possible that differences in PFC-mediated processing at encoding may additionally contribute to age-related memory deficits and/or the observed differences in retrieval activity. Future studies investigating both encoding and retrieval in the face of interference may allow us to better understand agerelated declines in overcoming interference.

\section{Conclusions}

The present study provides the first evidence that age-related associative memory impairments under conditions of high proactive interference are likely related to reduced engagement of DLPFC and anterior PFC-mediated post-retrieval monitoring and relational processes, respectively, but not reduced engagement of "lower-order" processes supported by the VLPFC. Collectively, these results suggest that the frontal lobe hypothesis likely oversimplifies the relationship between aging and the PFC (Kievit et al., 2014). Our results further suggest that the left mid-VLPFC may not serve a direct role in the selection of goal-relevant memory representations. Future work employing effective connectivity analyses may further answer exactly how these regions interact to resolve interference. Lastly, future studies that attempt to support resolution of interference during retrieval may be able to determine if the age-related alterations in the recruitment of strategic retrieval processes are intractable, or simply represent a failure to self-initiate these processes. 


\section{ACKNOWLEDGEMENTS}

We would like to acknowledge our participants for their time and contribution to this study. This material is based upon work supported by the National Science Foundation under Grant Number BCS-1125683 awarded to Audrey Duarte, as well as an APA Dissertation Award awarded to Michael Robert Dulas. 


\section{REFERENCES}

Achim, A. M., \& Lepage, M. (2005). Dorsolateral prefrontal cortex involvement in memory post-retrieval monitoring revealed in both item and associative recognition tests. Neuroimage, 24(4), 1113-1121.

Addis, D. R., Giovanello, K. S., Vu, M. A., \& Schacter, D. L. (2014). Age-related changes in prefrontal and hippocampal contributions to relational encoding. Neuroimage, 84, 19-26.

Ashburner, J. (2007). A fast diffeomorphic image registration algorithm. Neuroimage, 38(1), 95113.

Atkins, A. S., \& Reuter-Lorenz, P. A. (2011). Neural mechanisms of semantic interference and false recognition in short-term memory. Neuroimage, 56(3), 1726-1734.

Badre, D. (2008). Cognitive control, hierarchy, and the rostro-caudal organization of the frontal lobes. Trends Cogn Sci, 12(5), 193-200.

Badre, D., \& D'Esposito, M. (2007). Functional magnetic resonance imaging evidence for a hierarchical organization of the prefrontal cortex. J Cogn Neurosci, 19(12), 2082-2099.

Badre, D., \& D'Esposito, M. (2009). Is the rostro-caudal axis of the frontal lobe hierarchical? Nat Rev Neurosci, 10(9), 659-669.

Badre, D., Poldrack, R. A., Pare-Blagoev, E. J., Insler, R. Z., \& Wagner, A. D. (2005). Dissociable controlled retrieval and generalized selection mechanisms in ventrolateral prefrontal cortex. Neuron, 47(6), 907-918.

Badre, D., \& Wagner, A. D. (2007). Left ventrolateral prefrontal cortex and the cognitive control of memory. Neuropsychologia, 45(13), 2883-2901.

Barredo, J., Oztekin, I., \& Badre, D. (2013). Ventral Fronto-Temporal Pathway Supporting Cognitive Control of Episodic Memory Retrieval. Cereb Cortex, 10.1093/cercor/bht291.

Benton, A. L., Hamsher, S. K. d., \& Sivan, A. B. (1983). Multilingual aplasia examination (2nd ed.). Iowa City: AJA Associates.

Blumenfeld, R. S., \& Ranganath, C. (2007). Prefrontal cortex and long-term memory encoding: an integrative review of findings from neuropsychology and neuroimaging. Neuroscientist, 13(3), 280-291.

Bowles, R. P., \& Salthouse, T. A. (2003). Assessing the age-related effects of proactive interference on working memory tasks using the Rasch model. Psychol Aging, 18(3), 608-615.

Bowman, C. R., \& Dennis, N. A. (2015). Age differences in the neural correlates of novelty processing: The effects of item-relatedness. Brain Res, 1612, 2-15.

Bunge, S. A., Burrows, B., \& Wagner, A. D. (2004). Prefrontal and hippocampal contributions to visual associative recognition: interactions between cognitive control and episodic retrieval. Brain Cogn, 56(2), 141-152.

Bunge, S. A., Helskog, E. H., \& Wendelken, C. (2009). Left, but not right, rostrolateral prefrontal cortex meets a stringent test of the relational integration hypothesis. Neuroimage, 46(1), 338-342.

Burianova, H., Ciaramelli, E., Grady, C. L., \& Moscovitch, M. (2012). Top-down and bottom-up attention-to-memory: mapping functional connectivity in two distinct networks that underlie cued and uncued recognition memory. Neuroimage, 63(3), 1343-1352.

Cabeza, R. (2008). Role of parietal regions in episodic memory retrieval: the dual attentional processes hypothesis. Neuropsychologia, 46(7), 1813-1827. 
Cabeza, R., Anderson, N. D., Locantore, J. K., \& McIntosh, A. R. (2002). Aging gracefully: compensatory brain activity in high-performing older adults. Neuroimage, 17(3), 13941402.

Cansino, S., Hernandez-Ramos, E., \& Trejo-Morales, P. (2012). Neural correlates of source memory retrieval in young, middle-aged and elderly adults. Biol Psychol, 90(1), 33-49.

Chalfonte, B. L., \& Johnson, M. K. (1996). Feature memory and binding in young and older adults. Mem Cognit, 24(4), 403-416.

Christoff, K., Keramatian, K., Gordon, A. M., Smith, R., \& Madler, B. (2009). Prefrontal organization of cognitive control according to levels of abstraction. Brain Res, 1286, 94105.

Ciaramelli, E., Grady, C. L., \& Moscovitch, M. (2008). Top-down and bottom-up attention to memory: a hypothesis (AtoM) on the role of the posterior parietal cortex in memory retrieval. Neuropsychologia, 46(7), 1828-1851.

Daselaar, S. M., Fleck, M. S., Dobbins, I. G., Madden, D. J., \& Cabeza, R. (2006). Effects of healthy aging on hippocampal and rhinal memory functions: an event-related fMRI study. Cereb Cortex, 16(12), 1771-1782.

Davis, S. W., Dennis, N. A., Daselaar, S. M., Fleck, M. S., \& Cabeza, R. (2008). Que PASA? The posterior-anterior shift in aging. Cereb Cortex, 18(5), 1201-1209.

Dennis, N. A., Kim, H., \& Cabeza, R. (2008). Age-related Differences in Brain Activity during True and False Memory Retrieval. J Cogn Neurosci, 10.1162/jocn.2008.20096.

Diana, R. A., Yonelinas, A. P., \& Ranganath, C. (2007). Imaging recollection and familiarity in the medial temporal lobe: a three-component model. Trends Cogn Sci, 11(9), 379-386.

Diana, R. A., Yonelinas, A. P., \& Ranganath, C. (2009). Medial Temporal Lobe Activity during Source Retrieval Reflects Information Type, not Memory Strength. J Cogn Neurosci, 10.1162/jocn.2009.21335.

Dobbins, I. G., Foley, H., Schacter, D. L., \& Wagner, A. D. (2002). Executive control during episodic retrieval: multiple prefrontal processes subserve source memory. Neuron, 35(5), 989-996.

Dobbins, I. G., \& Han, S. (2006). Isolating rule- versus evidence-based prefrontal activity during episodic and lexical discrimination: a functional magnetic resonance imaging investigation of detection theory distinctions. Cereb Cortex, 16(11), 1614-1622.

Dobbins, I. G., Rice, H. J., Wagner, A. D., \& Schacter, D. L. (2003). Memory orientation and success: separable neurocognitive components underlying episodic recognition. Neuropsychologia, 41(3), 318-333.

Domenech, P., \& Koechlin, E. (2015). Executive control and decision-making in the prefrontal cortex. Current Opinion in Behavioral Scienes, 1, 101-106.

Donaldson, D. I., Wheeler, M. E., \& Petersen, S. E. (2010). Remember the source: dissociating frontal and parietal contributions to episodic memory. J Cogn Neurosci, 22(2), 377-391.

Duarte, A., Graham, K. S., \& Henson, R. N. (2010). Age-related changes in neural activity associated with familiarity, recollection and false recognition. Neurobiol Aging, 31(10), 1814-1830.

Duarte, A., Henson, R. N., \& Graham, K. S. (2008). The effects of aging on the neural correlates of subjective and objective recollection. Cereb Cortex, 18(9), 2169-2180.

Dulas, M. R., \& Duarte, A. (2011). The effects of aging on material-independent and materialdependent neural correlates of contextual binding. Neuroimage, 57(3), 1192-1204. 
Dulas, M. R., \& Duarte, A. (2012). The effects of aging on material-independent and materialdependent neural correlates of source memory retrieval. Cereb Cortex, 22(1), 37-50.

Dulas, M. R., \& Duarte, A. (2013). The influence of directed attention at encoding on source memory retrieval in the young and old: an ERP study. Brain Res.

Dulas, M. R., \& Duarte, A. (2014). Aging Affects the Interaction between Attentional Control and Source Memory: An fMRI Study. J Cogn Neurosci, 10.1162/jocn_a_00663.

Dulas, M. R., Newsome, R. N., \& Duarte, A. (2011). The effects of aging on ERP correlates of source memory retrieval for self-referential information. Brain Res, 1377, 84-100.

Duverne, S., Habibi, A., \& Rugg, M. D. (2008). Regional specificity of age effects on the neural correlates of episodic retrieval. Neurobiol Aging, 29(12), 1902-1916.

Duverne, S., Motamedinia, S., \& Rugg, M. D. (2009). The relationship between aging, performance, and the neural correlates of successful memory encoding. Cereb Cortex, 19(3), 733-744.

Duzel, E., Schutze, H., Yonelinas, A. P., \& Heinze, H. J. (2011). Functional phenotyping of successful aging in long-term memory: Preserved performance in the absence of neural compensation. Hippocampus, 21(8), 803-814.

Ebner, N. C., Riediger, M., \& Lindenberger, U. (2010). FACES--a database of facial expressions in young, middle-aged, and older women and men: development and validation. Behav Res Methods, 42(1), 351-362.

Eichenbaum, H., Yonelinas, A. P., \& Ranganath, C. (2007). The medial temporal lobe and recognition memory. Annu Rev Neurosci, 30, 123-152.

Fandakova, Y., Lindenberger, U., \& Shing, Y. L. (2013). Deficits in Process-Specific Prefrontal and Hippocampal Activations Contribute to Adult Age Differences in Episodic Memory Interference. Cereb Cortex, 10.1093/cercor/bht034.

Feredoes, E., Heinen, K., Weiskopf, N., Ruff, C., \& Driver, J. (2011). Causal evidence for frontal involvement in memory target maintenance by posterior brain areas during distracter interference of visual working memory. Proc Natl Acad Sci U S A, 108(42), 1751017515.

Fletcher, P. C., \& Henson, R. N. (2001). Frontal lobes and human memory: insights from functional neuroimaging. Brain, 124(Pt 5), 849-881.

Friedman, D. (2000). Event-related brain potential investigations of memory and aging. Biol Psychol, 54(1-3), 175-206.

Glisky, E. L., \& Kong, L. L. (2008). Do young and older adults rely on different processes in source memory tasks? A neuropsychological study. J Exp Psychol Learn Mem Cogn, 34(4), 809-822.

Glisky, E. L., Rubin, S. R., \& Davidson, P. S. (2001). Source memory in older adults: an encoding or retrieval problem? J Exp Psychol Learn Mem Cogn, 27(5), 1131-1146.

Gold, B. T., Balota, D. A., Jones, S. J., Powell, D. K., Smith, C. D., \& Andersen, A. H. (2006). Dissociation of automatic and strategic lexical-semantics: functional magnetic resonance imaging evidence for differing roles of multiple frontotemporal regions. J Neurosci, 26(24), 6523-6532.

Grady, C. (2012). The cognitive neuroscience of ageing. Nat Rev Neurosci, 13(7), 491-505.

Grady, C. L., McIntosh, A. R., \& Craik, F. I. (2005). Task-related activity in prefrontal cortex and its relation to recognition memory performance in young and old adults. Neuropsychologia, 43(10), 1466-1481. 
Han, S., Huettel, S. A., \& Dobbins, I. G. (2009). Rule-dependent prefrontal cortex activity across episodic and perceptual decisions: an fMRI investigation of the criterial classification account. J Cogn Neurosci, 21(5), 922-937.

Han, S., O'Connor, A. R., Eslick, A. N., \& Dobbins, I. G. (2012). The role of left ventrolateral prefrontal cortex during episodic decisions: semantic elaboration or resolution of episodic interference? J Cogn Neurosci, 24(1), 223-234.

Hasher, L., \& Zacks, R. (1979). Automatic and effortful processes in memory. Journal of Experimental Psychology: General, 108, 356-388.

Hay, J. F., \& Jacoby, L. L. (1999). Separating habit and recollection in young and older adults: effects of elaborative processing and distinctiveness. Psychol Aging, 14(1), 122-134.

Hayes, S. M., Buchler, N., Stokes, J., Kragel, J., \& Cabeza, R. (2011). Neural correlates of confidence during item recognition and source memory retrieval: evidence for both dualprocess and strength memory theories. J Cogn Neurosci, 23(12), 3959-3971.

Hazeltine, E., Bunge, S. A., Scanlon, M. D., \& Gabrieli, J. D. (2003). Material-dependent and material-independent selection processes in the frontal and parietal lobes: an eventrelated fMRI investigation of response competition. Neuropsychologia, 41(9), 12081217.

Hedden, T., \& Gabrieli, J. D. (2005). Healthy and pathological processes in adult development: new evidence from neuroimaging of the aging brain. Curr Opin Neurol, 18(6), 740-747.

Henson, R. N. (2007). Efficient Experimental Design for fMRI. In J. T. Ashburner, S. J. Kiebel, T. E. Nichols \& W. D. Penny (Eds.), Statistical parametric mapping. The analysis of functional brain images (pp. 193-210). London: Academic Press.

Henson, R. N., Rugg, M. D., Shallice, T., \& Dolan, R. J. (2000). Confidence in recognition memory for words: dissociating right prefrontal roles in episodic retrieval. J Cogn Neurosci, 12(6), 913-923.

Henson, R. N., Rugg, M. D., Shallice, T., Josephs, O., \& Dolan, R. J. (1999). Recollection and familiarity in recognition memory: an event-related functional magnetic resonance imaging study. J Neurosci, 19(10), 3962-3972.

Henson, R. N., Shallice, T., \& Dolan, R. J. (1999). Right prefrontal cortex and episodic memory retrieval: a functional MRI test of the monitoring hypothesis. Brain, 122 ( Pt 7), 13671381.

Jacoby, L. L., Bishara, A. J., Hessels, S., \& Toth, J. P. (2005). Aging, subjective experience, and cognitive control: dramatic false remembering by older adults. J Exp Psychol Gen, 134(2), 131-148.

Jacoby, L. L., Wahlheim, C. N., Rhodes, M. G., Daniels, K. A., \& Rogers, C. S. (2010). Learning to diminish the effects of proactive interference: reducing false memory for young and older adults. Mem Cognit, 38(6), 820-829.

Johnson, M. K., Hashtroudi, S., \& Lindsay, D. S. (1993). Source monitoring. Pschol. Rev., 114, 3-28.

Jonides, J., Marshuetz, C., Smith, E. E., Reuter-Lorenz, P. A., Koeppe, R. A., \& Hartley, A. (2000). Age differences in behavior and PET activation reveal differences in interference resolution in verbal working memory. J Cogn Neurosci, 12(1), 188-196.

Jonides, J., \& Nee, D. E. (2006). Brain mechanisms of proactive interference in working memory. Neuroscience, 139(1), 181-193. 
Kievit, R. A., Davis, S. W., Mitchell, D. J., Taylor, J. R., Duncan, J., Cam, C. A. N. R. T., . . Cam, C. A. N. R. T. (2014). Distinct aspects of frontal lobe structure mediate age-related differences in fluid intelligence and multitasking. Nat Commun, 5, 5658.

Kim, C., Johnson, N. F., \& Gold, B. T. (2012). Common and distinct neural mechanisms of attentional switching and response conflict. Brain Res, 1469, 92-102.

Koutstaal, W., \& Schacter, D. L. (1997). Gist-based false recognition of pictures in older and younger adults. Journal of Memory and Language, 37, 555-583.

Kuhl, B. A., Bainbridge, W. A., \& Chun, M. M. (2012). Neural reactivation reveals mechanisms for updating memory. J Neurosci, 32(10), 3453-3461.

Leshikar, E. D., Gutchess, A. H., Hebrank, A. C., Sutton, B. P., \& Park, D. C. (2010). The impact of increased relational encoding demands on frontal and hippocampal function in older adults. Cortex, 46(4), 507-521.

Li, J., Morcom, A. M., \& Rugg, M. D. (2004). The effects of age on the neural correlates of successful episodic retrieval: an ERP study. Cogn Affect Behav Neurosci, 4(3), 279-293.

Logan, J. M., Sanders, A. L., Snyder, A. Z., Morris, J. C., \& Buckner, R. L. (2002). Underrecruitment and nonselective recruitment: dissociable neural mechanisms associated with aging. Neuron, 33(5), 827-840.

Luo, L., \& Craik, F. I. (2008). Aging and memory: a cognitive approach. Can J Psychiatry, 53(6), 346-353.

Lustig, C., \& Jantz, T. (2015). Questions of age differences in interference control: When and how, not if? Brain Res, 1612, 59-69.

Lustig, C., May, C. P., \& Hasher, L. (2001). Working memory span and the role of proactive interference. J Exp Psychol Gen, 130(2), 199-207.

MacPherson, S. E., Phillips, L. H., \& Della Sala, S. (2002). Age, executive function, and social decision making: a dorsolateral prefrontal theory of cognitive aging. Psychol Aging, 17(4), 598-609.

May, C. P., Hasher, L., \& Kane, M. J. (1999). The role of interference in memory span. Mem Cognit, 27(5), 759-767.

McDonough, I. M., Wong, J. T., \& Gallo, D. A. (2012). Age-Related Differences in Prefrontal Cortex Activity during Retrieval Monitoring: Testing the Compensation and Dysfunction Accounts. Cereb Cortex, 10.1093/cercor/bhs064.

Mitchell, K. J., \& Johnson, M. K. (2009). Source monitoring 15 years later: what have we learned from fMRI about the neural mechanisms of source memory? Psychol Bull, 135(4), 638-677.

Morcom, A. M., Li, J., \& Rugg, M. D. (2007). Age effects on the neural correlates of episodic retrieval: increased cortical recruitment with matched performance. Cereb Cortex, $17(11), 2491-2506$.

Naveh-Benjamin, M. (2000). Adult age differences in memory performance: tests of an associative deficit hypothesis. J Exp Psychol Learn Mem Cogn, 26(5), 1170-1187.

Naveh-Benjamin, M., Brav, T. K., \& Levy, O. (2007). The associative memory deficit of older adults: the role of strategy utilization. Psychol Aging, 22(1), 202-208.

Nee, D. E., Wager, T. D., \& Jonides, J. (2007). Interference resolution: insights from a metaanalysis of neuroimaging tasks. Cogn Affect Behav Neurosci, 7(1), 1-17.

Nelson, J. K., Reuter-Lorenz, P. A., Persson, J., Sylvester, C. Y., \& Jonides, J. (2009). Mapping interference resolution across task domains: a shared control process in left inferior frontal gyrus. Brain Res, 1256, 92-100. 
Nelson, J. K., Reuter-Lorenz, P. A., Sylvester, C. Y., Jonides, J., \& Smith, E. E. (2003). Dissociable neural mechanisms underlying response-based and familiarity-based conflict in working memory. Proc Natl Acad Sci U S A, 100(19), 11171-11175.

Norman, K. A., \& Schacter, D. L. (1997). False recognition in younger and older adults: exploring the characteristics of illusory memories. Mem Cognit, 25(6), 838-848.

Ochsner, K. N., Hughes, B., Robertson, E. R., Cooper, J. C., \& Gabrieli, J. D. (2009). Neural systems supporting the control of affective and cognitive conflicts. J Cogn Neurosci, 21(9), 1842-1855.

Old, S. R., \& Naveh-Benjamin, M. (2008). Differential effects of age on item and associative measures of memory: a meta-analysis. Psychol Aging, 23(1), 104-118.

Oztekin, I., Curtis, C. E., \& McElree, B. (2009). The medial temporal lobe and the left inferior prefrontal cortex jointly support interference resolution in verbal working memory. $J$ Cogn Neurosci, 21(10), 1967-1979.

Pereira, J. M., Xiong, L., Acosta-Cabronero, J., Pengas, G., Williams, G. B., \& Nestor, P. J. Registration accuracy for VBM studies varies according to region and degenerative disease grouping. Neuroimage, 49(3), 2205-2215.

Rajah, M. N., \& D'Esposito, M. (2005). Region-specific changes in prefrontal function with age: a review of PET and fMRI studies on working and episodic memory. Brain, 128(Pt 9), 1964-1983.

Rajah, M. N., Languay, R., \& Valiquette, L. (2010). Age-related changes in prefrontal cortex activity are associated with behavioural deficits in both temporal and spatial context memory retrieval in older adults. Cortex, 46(4), 535-549.

Ranganath, C., Heller, A. S., \& Wilding, E. L. (2007). Dissociable correlates of two classes of retrieval processing in prefrontal cortex. Neuroimage, 35(4), 1663-1673.

Raz, N., Gunning, F. M., Head, D., Dupuis, J. H., McQuain, J., Briggs, S. D., . . Acker, J. D. (1997). Selective aging of the human cerebral cortex observed in vivo: differential vulnerability of the prefrontal gray matter. Cereb Cortex, 7(3), 268-282.

Rugg, M. D., Johnson, J. D., Park, H., \& Uncapher, M. R. (2008). Encoding-retrieval overlap in human episodic memory: a functional neuroimaging perspective. Prog Brain Res, 169, 339-352.

Rugg, M. D., \& Morcom, A. M. (2005). The relationship between brain activity, cognitive performance and aging: the case of memory. In R. Cabeza, L. Nyberg \& D. C. Park (Eds.), Cognitive Neuroscience of Aging (pp. 132-154). Oxford: Oxford University Press.

Rypma, B., \& D'Esposito, M. (2000). Isolating the neural mechanisms of age-related changes in human working memory. Nat Neurosci, 3(5), 509-515.

Rypma, B., Eldreth, D. A., \& Rebbechi, D. (2007). Age-related differences in activationperformance relations in delayed-response tasks: a multiple component analysis. Cortex, 43(1), 65-76.

Slotnick, S. D., Moo, L. R., Segal, J. B., \& Hart, J., Jr. (2003). Distinct prefrontal cortex activity associated with item memory and source memory for visual shapes. Brain Res Cogn Brain Res, 17(1), 75-82.

Snodgrass, J., \& Corwin, J. (1988). Pragmatics of measuring recognition memory: applications to dementia and amnesia. Journal of Experimental Psychology, 116, 34-50.

Staresina, B. P., Henson, R. N., Kriegeskorte, N., \& Alink, A. (2012). Episodic reinstatement in the medial temporal lobe. J Neurosci, 32(50), 18150-18156. 
Swick, D., Senkfor, A. J., \& Van Petten, C. (2006). Source memory retrieval is affected by aging and prefrontal lesions: behavioral and ERP evidence. Brain Res, 1107(1), 161-176.

Thompson-Schill, S. L., Bedny, M., \& Goldberg, R. F. (2005). The frontal lobes and the regulation of mental activity. Curr Opin Neurobiol, 15(2), 219-224.

Thompson-Schill, S. L., Jonides, J., Marshuetz, C., Smith, E. E., D'Esposito, M., Kan, I. P., . . . Swick, D. (2002). Effects of frontal lobe damage on interference effects in working memory. Cogn Affect Behav Neurosci, 2(2), 109-120.

Turner, M. S., Simons, J. S., Gilbert, S. J., Frith, C. D., \& Burgess, P. W. (2008). Distinct roles for lateral and medial rostral prefrontal cortex in source monitoring of perceived and imagined events. Neuropsychologia, 46(5), 1442-1453.

Ullsperger, M., \& von Cramon, D. Y. (2001). Subprocesses of performance monitoring: a dissociation of error processing and response competition revealed by event-related fMRI and ERPs. Neuroimage, 14(6), 1387-1401.

van Veen, V., \& Carter, C. S. (2002). The anterior cingulate as a conflict monitor: fMRI and ERP studies. Physiol Behav, 77(4-5), 477-482.

Vilberg, K. L., \& Rugg, M. D. (2008). Memory retrieval and the parietal cortex: A review of evidence from a dual-process perspective. Neuropsychologia, 46(7), 1787-1799.

West, R. L. (1996). An application of prefrontal cortex function theory to cognitive aging. Psychol Bull, 120(2), 272-292.

Williams, J. (1991). Memory assessment scales professional manual. Odessa: Psychological Assessment Resources.

Xiao, J., Hays, J., Ehinger, K., Oliva, A., \& Torralba, A. (2010). SUN Database: Large-scale Scene Recognition from Abbey to Zoo. IEEE Conference on Computer Vision and Pattern Recognition.

Yassa, M. A., \& Stark, C. E. (2009). A quantitative evaluation of cross-participant registration techniques for MRI studies of the medial temporal lobe. Neuroimage, 44(2), 319-327.

Yonelinas, A. P., \& Jacoby, L. L. (2012). The process-dissociation approach two decades later: convergence, boundary conditions, and new directions. Mem Cognit, 40(5), 663-680.

Yonelinas, A. P., Otten, L. J., Shaw, K. N., \& Rugg, M. D. (2005). Separating the brain regions involved in recollection and familiarity in recognition memory. J Neurosci, 25(11), 30023008.

Zeithamova, D., \& Preston, A. R. (2010). Flexible memories: differential roles for medial temporal lobe and prefrontal cortex in cross-episode binding. J Neurosci, 30(44), 1467614684. 
Table 1. Group characteristics.

\begin{tabular}{lcc}
\hline Measure & $\begin{array}{c}\text { Young } \\
(\mathrm{n}=22)\end{array}$ & $\begin{array}{c}\text { Old } \\
(\mathrm{n}=22)\end{array}$ \\
\hline Age & $24.41(4.65)$ & $67.90(4.61)$ \\
Gender & $15 / 22$ female & $12 / 22$ female \\
Education & $16.34(2.34)$ & $16.55(2.28)$ \\
Letter Fluency & $49.64(8.93)$ & $57.00(14.04)$ \\
List Recall (Immediate) & $10.77(1.45)$ & $10.41(1.53)$ \\
List Recall (Immediate, Cued) & $10.95(1.21)$ & $10.59(1.26)$ \\
List Recall (Delayed) & $11.45(0.67)$ & $11.18(1.40)$ \\
List Recall (Delayed, Cued) & $11.23(1.93)$ & $11.50(0.91)$ \\
List Recognition & $12.00(0.00)$ & $11.95(0.21)$ \\
MAS Digit Span Forward & $6.82(1.33)$ & $6.55(1.44)$ \\
MAS Digit Span Backward & $4.73(1.08)$ & $4.73(1.61)$ \\
Trails A (in seconds) & $23.78(5.46)$ & $35.42(10.62)^{*}$ \\
Trails B (in seconds) & $47.78(15.22)$ & $61.35(21.93)^{*}$ \\
Visual Recognition & $19.05(1.53)$ & $16.86(2.27)^{*}$ \\
Delayed Visual Recognition & $19.23(1.02)$ & $17.14(2.66)^{*}$ \\
Visual Reproduction & $8.36(1.97)$ & $4.82(2.06)^{*}$
\end{tabular}

Note: Standard deviations in parentheses. All neuropsychological test scores are reported as raw scores. $*=$ significantly worse than Young $(\mathrm{p}<0.05)$. 
Table 2. Response proportions, performances indices, and response times to studied and unstudied objects at test.

\begin{tabular}{|c|c|c|c|c|}
\hline & \multicolumn{2}{|c|}{ Young Adults } & \multicolumn{2}{|c|}{ Older Adults } \\
\hline & High & Low & High & Low \\
\hline & Interference & Interference & Interference & Interference \\
\hline \multicolumn{5}{|l|}{ Response proportions } \\
\hline \multicolumn{5}{|l|}{ Studied Objects } \\
\hline Correct Associate & $0.63(0.16)$ & $0.84(0.09)$ & $0.55(0.11)$ & $0.77(0.13)$ \\
\hline Incorrect Associate & $0.34(0.14)$ & $0.12(0.05)$ & $0.38(0.07)$ & $0.16(0.08)$ \\
\hline Miss (New Response) & $0.03(0.03)$ & $0.04(0.04)$ & $0.07(0.07)$ & $0.07(0.07)$ \\
\hline \multicolumn{5}{|l|}{ Unstudied Objects } \\
\hline Correct Rejections (CR) & \multicolumn{2}{|c|}{$0.97(0.03)$} & \multicolumn{2}{|c|}{$0.95(0.06)$} \\
\hline \multicolumn{5}{|l|}{ Associative proportions of hits } \\
\hline $\mathrm{P}($ correct associate $)$ & $0.65(0.15)$ & $0.87(0.06)$ & $0.58(0.09)$ & $0.83(0.09)$ \\
\hline $\mathrm{P}($ incorrect associate $)$ & $0.35(0.15)$ & $0.13(0.06)$ & $0.42(0.09)$ & $0.17(0.09)$ \\
\hline \multicolumn{5}{|l|}{ Performance Indices } \\
\hline Item Memory Accuracy (Pr) & $0.95(0.04)$ & $0.94(0.05)$ & $0.90(0.02)$ & $0.91(0.02)$ \\
\hline Associative Memory Accuracy & $0.65(0.15)$ & $0.87(0.06)$ & $0.58(0.09)$ & $0.83(0.09)$ \\
\hline \multicolumn{5}{|l|}{ Response Times } \\
\hline \multicolumn{5}{|l|}{ Associative Memory Response } \\
\hline Correct Associate & $969(302)$ & $870(270)$ & $1267(337)$ & $1171(337)$ \\
\hline Incorrect Associate & $1084(361)$ & $1284(416)$ & $1334(326)$ & $1500(386)$ \\
\hline
\end{tabular}


Table 3. Regions showing age-related differences in old-new effects across interference conditions.

\begin{tabular}{|c|c|c|c|c|c|c|}
\hline Contrast & Region & $\mathrm{L} / \mathrm{R}$ & $\begin{array}{c}\text { MNI } \\
\text { Coordinates } \\
(\mathrm{x}, \mathrm{y}, \mathrm{z})\end{array}$ & BA & T score & $\begin{array}{c}\text { Cluster } \\
\text { Size }\end{array}$ \\
\hline \multicolumn{7}{|l|}{$\mathrm{AC}>\mathbf{C R}$} \\
\hline \multicolumn{7}{|c|}{ Young $>$ Old } \\
\hline & Middle Frontal Gyrus & $\mathrm{R}$ & $48,32,36$ & $45 / 46$ & 3.92 & 130 \\
\hline & Ventral Parietal Cortex & $\mathrm{R}$ & $54,-57,45$ & 39 & 3.59 & 30 \\
\hline & Orbitofrontal Cortex & $\mathrm{R}$ & $41,56,-5$ & 46 & 3.55 & 45 \\
\hline \multicolumn{7}{|c|}{ Old $>$ Young } \\
\hline & Ventral Parietal Cortex (Angular Gyrus) & $\mathrm{L}$ & $-38,-70,42$ & $7 / 19$ & 4.68 & 104 \\
\hline & Middle Frontal Gyrus & $\mathrm{L}$ & $-27,17,52$ & 8 & 4.66 & 262 \\
\hline & Middle Cingulate & $\mathrm{L}$ & $-3,-33,40$ & 23 & 4.53 & 169 \\
\hline & Middle Occipital Cortex & $\mathrm{L}$ & $-38,-82,28$ & 19 & 4.15 & 235 \\
\hline & & $\mathrm{R}$ & $30,-76,24$ & 19 & 3.47 & 46 \\
\hline
\end{tabular}

$\mathrm{L}=$ Left; $\mathrm{R}=$ Right; BA = Broadmann's area; $\mathrm{AC}=$ Associative Correct; $\mathrm{CR}=$ Correct Rejection. 
Table 4. Regions showing interference related effects.

\begin{tabular}{|c|c|c|c|c|c|c|}
\hline Contrast & Region & $\mathrm{L} / \mathrm{R}$ & $\begin{array}{c}\text { MNI } \\
\text { Coordinates } \\
(\mathrm{x}, \mathrm{y}, \mathrm{z}) \\
\end{array}$ & BA & $\begin{array}{c}\mathrm{T} \\
\text { Score }\end{array}$ & $\begin{array}{l}\text { Cluster } \\
\text { Size }\end{array}$ \\
\hline \multicolumn{7}{|l|}{ High AC $>$ Low AC } \\
\hline \multicolumn{7}{|c|}{ Across Groups } \\
\hline & Middle Cingulate & $\mathrm{R}$ & $10,26,34$ & 32 & 4.31 & 78 \\
\hline & Middle Cingulate & $\mathrm{L}$ & $-6,21,40$ & 32 & 3.82 & 150 \\
\hline & Anterior Cingulate & $L$ & $-9,27,30$ & 32 & 3.70 & \\
\hline & Precentral Gyrus & $\mathrm{L}$ & $-36,-1,40$ & 6 & 3.92 & 70 \\
\hline & Supplemental Motor Area & $\mathrm{L}$ & $-10,9,58$ & 6 & 4.06 & 189 \\
\hline & Superior Frontal Gyrus & $\mathrm{L}$ & $-21,-1,60$ & 6 & 3.65 & 35 \\
\hline & Ventrolateral Prefrontal Cortex & $\mathbf{L}$ & $-50,24,31$ & $44 / 45$ & 3.29 & 37 \\
\hline & Parahippocampal Cortex & $\mathrm{L}$ & $-24,-36,-11$ & 30 & 3.69 & 34 \\
\hline & Superior Parietal Cortex & $\mathbf{L}$ & $-30,-48,39$ & 40 & 3.64 & 42 \\
\hline & Middle Occipital Cortex & $\mathrm{L}$ & $-21,-99,1$ & 18 & 3.61 & 40 \\
\hline & Vermis & B & $-2,-46,-18$ & & 4.92 & 97 \\
\hline & Caudate & $\mathrm{R}$ & $16,17,3$ & & 4.34 & 300 \\
\hline
\end{tabular}

L = Left; R = Right; B = Bilateral; BA = Broadmann's area; AC = Associative Correct. Italicized regions represent sub-clusters. Regions in bold indicate regions show in Figures 3. 
Table 5. Regions showing associative memory accuracy effects for high interference trials.

\begin{tabular}{|c|c|c|c|c|c|c|}
\hline Contrast & Region & $\mathrm{L} / \mathrm{R}$ & $\begin{array}{c}\text { MNI } \\
\text { Coordinates } \\
(\mathrm{x}, \mathrm{y}, \mathrm{z}) \\
\end{array}$ & $\mathrm{BA}$ & $\begin{array}{c}\mathrm{T} \\
\text { Score } \\
\end{array}$ & $\begin{array}{c}\text { Cluste } \\
\text { Size } \\
\end{array}$ \\
\hline \multicolumn{7}{|l|}{$\mathbf{A C}>\mathbf{A I}$} \\
\hline \multicolumn{7}{|c|}{ Across Groups } \\
\hline & Hippocampus & $\mathbf{L}$ & $-20,-7,-12$ & & 4.16 & 56 \\
\hline & Paracentral Lobule & $\mathrm{L}$ & $-14,-24,64$ & 4 & 4.15 & 157 \\
\hline & Precentral Gyrus & $\mathrm{L}$ & $-42,-7,42$ & 6 & 3.86 & 49 \\
\hline & Supplemental Motor Area & $\mathrm{R}$ & $12,-12,70$ & 6 & 3.59 & 40 \\
\hline & Superior Frontal Gyrus & $\mathrm{L}$ & $-20,-3,69$ & 6 & 3.54 & 30 \\
\hline \multicolumn{7}{|c|}{ Young $>$ Old } \\
\hline & Anterior Prefrontal Cortex & $\mathbf{L}$ & $-9,65,19$ & 10 & 3.65 & 38 \\
\hline & & $\mathrm{R}$ & $12,65,13$ & 10 & 3.61 & 41 \\
\hline & Dorsolateral Prefrontal Cortex & $\mathbf{L}$ & $-22,39,49$ & 9 & 3.39 & 34 \\
\hline & Middle Temporal Cortex & $\mathrm{L}$ & $-55,-12,-15$ & 20 & 3.78 & 64 \\
\hline & Precentral Gyrus & $\mathrm{R}$ & $16,-30,66$ & 4 & 3.57 & 127 \\
\hline & Middle Cingulate & $\mathrm{R}$ & $6,-24,49$ & & 4.27 & 137 \\
\hline & Supplemental Motor Area & $\mathrm{R}$ & $8,9,63$ & 6 & 3.96 & 73 \\
\hline & & $\mathrm{L}$ & $-12,-9,70$ & 6 & 3.72 & 274 \\
\hline & Cerebellum & $\mathrm{L}$ & $-38,-78,-30$ & & 3.83 & 36 \\
\hline
\end{tabular}

L = Left; R = Right; BA = Broadmann's area; $\mathrm{AC}=$ Associative Correct; $\mathrm{AI}=$ Associative Incorrect. Regions in bold indicate regions shown in Figures 4 and 5. 


\section{LIST OF FIGURES}

Figure 1. Encoding Design (not scanned). Examples of each condition are shown, the numbers indicating the number of times (across blocks) each object is shown with either the lure or the critical (most recent) associate. E.g. for High interference, the critical (last) pairing was only shown twice and the interfering pairing was shown the first 3 times.

Figure 2. Retrieval Design (scanned). Examples corresponding to the previous figure are shown.

Figure 3. Interference Effects. High > Low Interference effects at test for selected regions are displayed on MNI reference brains. Plots show parameter estimates of for High Interference Associative Correct, Low Interference Associative Correct, and Correct Rejection trials for both groups. There was no true baseline for this study (i.e. fixation trials), thus the zero line of the $\mathrm{x}$ axis cannot be interpreted as a baseline. Therefore, any "activations" or "deactivations" relative to the zero line are more apparent than real. Only the contrasts between conditions are interpretable. Error bars depict standard error of the mean across participants for each group [ $p<$ 0.001, uncorrected, with a 17 voxel extent; exclusive masking conducted as described in section

\section{6 fMRI Analysis].}

Figure 4. Associative Memory Accuracy Effects. Associative memory accuracy effects for selected regions are displayed on MNI reference brains. Plots show parameter estimates of Associative Correct trials and Associative Incorrect trials for only High Interference. Baseline, thresholds, and standard errors as in Figure 3.

Figure 5. Age-related Associative Memory Accuracy Effects. Associative memory accuracy effects for selected regions are displayed on MNI reference brains. Plots show parameter estimates of Associative Correct trials and Associative Incorrect trials for only High Interference. Baseline, thresholds, and standard errors as in Figure 3. 
Figure 6. Left VLPFC Sensitive to Interference, not Accuracy. High Interference Associative Correct > Low Interference Associative Correct effects for selected region are displayed on MNI reference brains. Plots show parameter estimates of trials for High Interference Associative Correct, High Interference Associative Incorrect, and Low Interference Associative Correct for both groups. Baseline, thresholds, and standard errors as in Figure 3. 


\section{Encoding Design Low Interference}

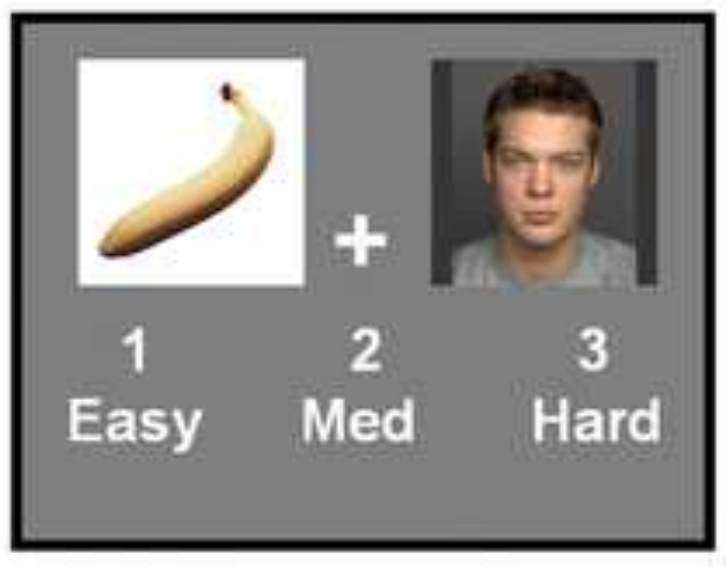

Lure $\times 1$

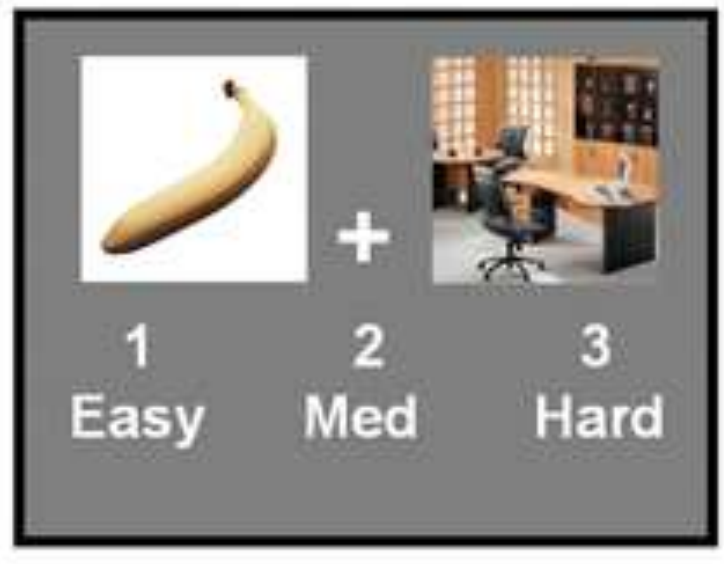

Target $\times 4$

High Interference

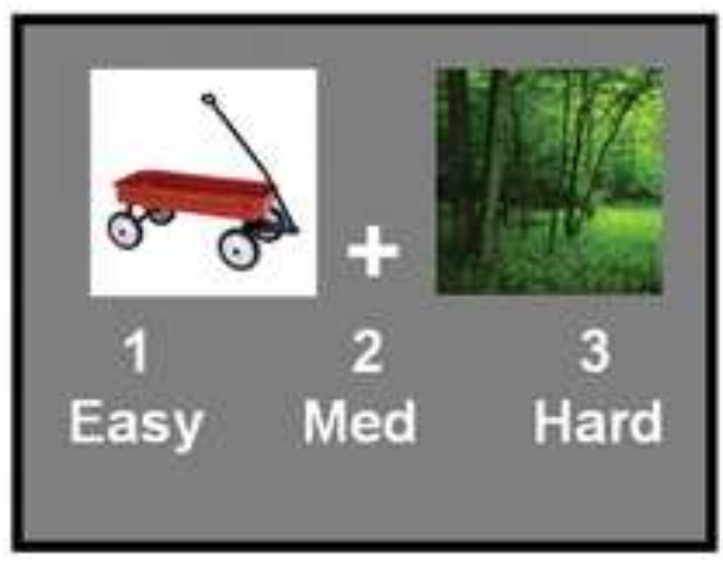

Lure $\times 3$

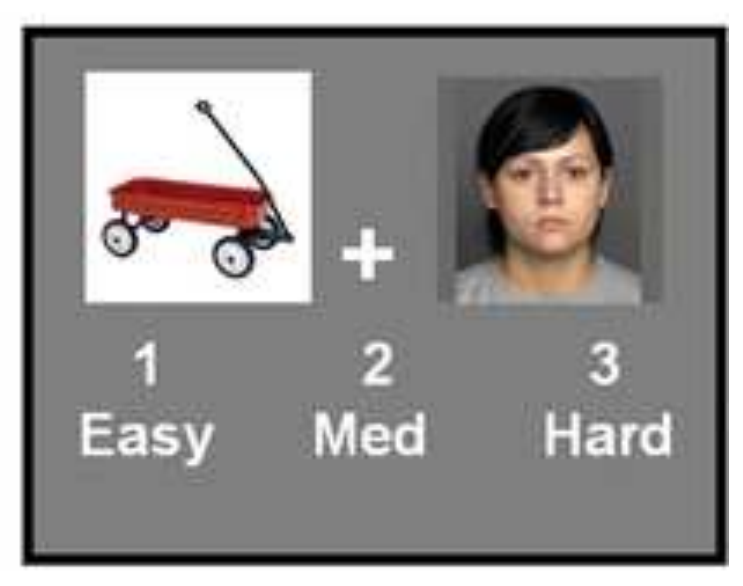

Target $\times 2$ 


\section{Figure 2}

\section{Retrieval Design

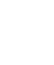

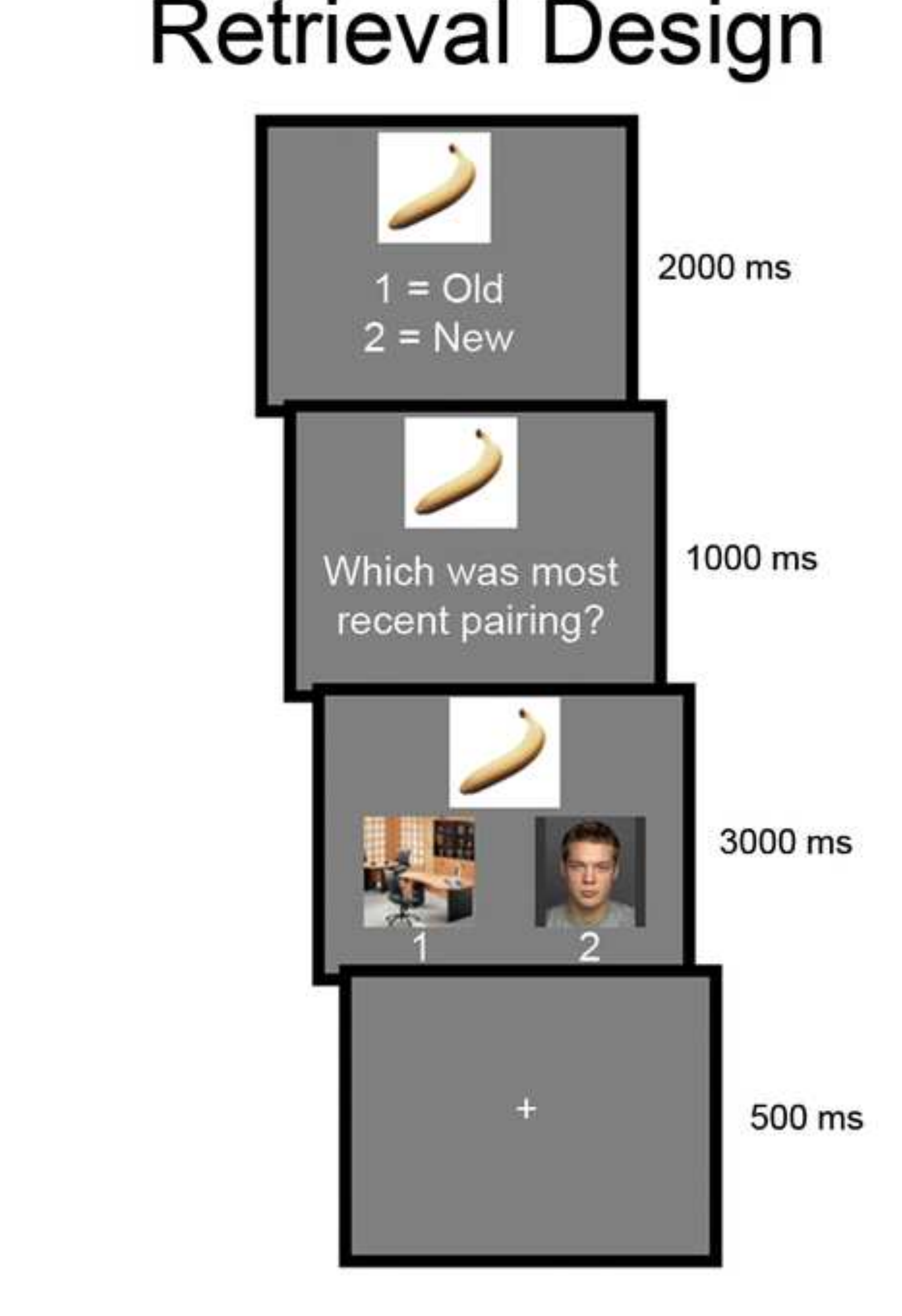

$500 \mathrm{~ms}$

列

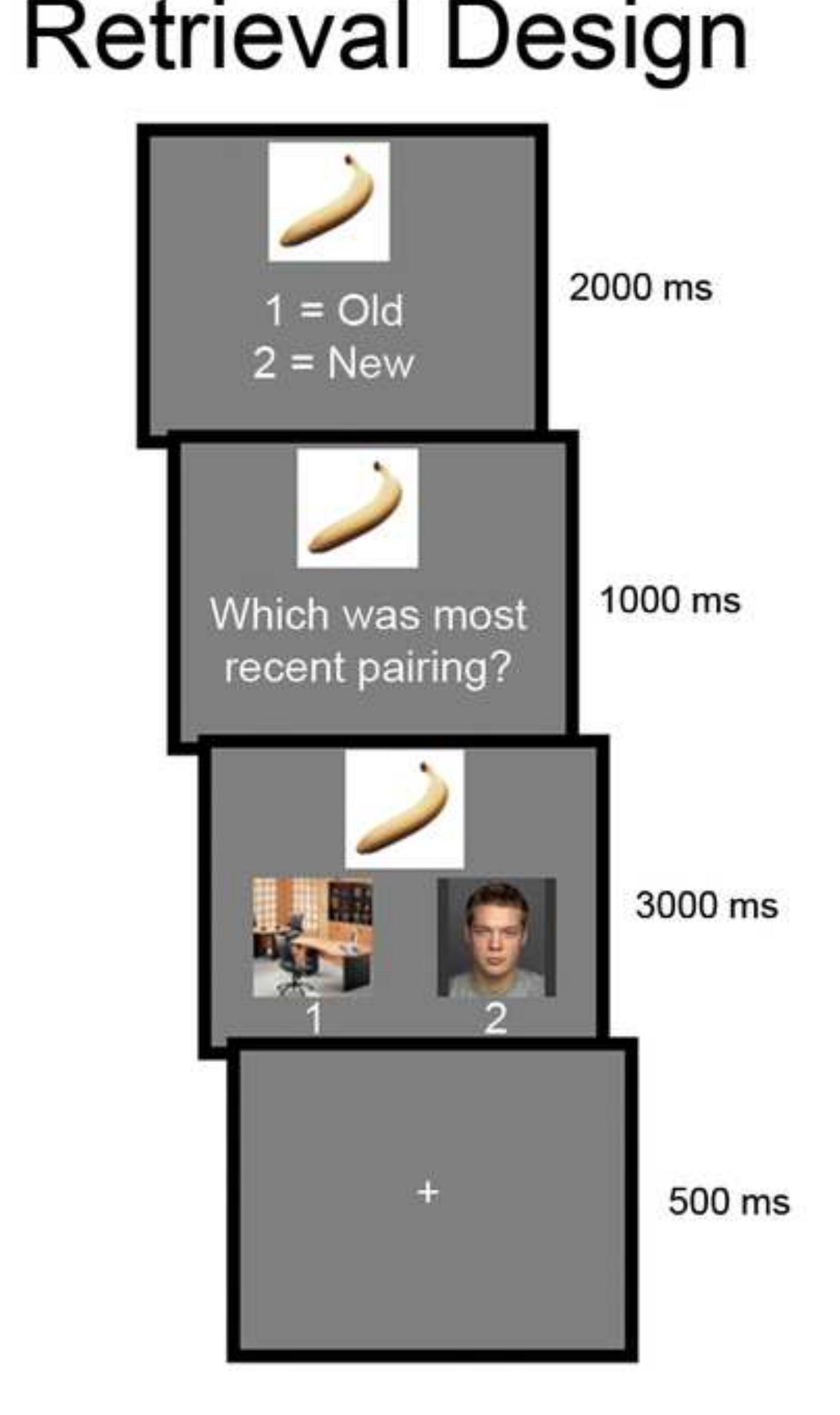

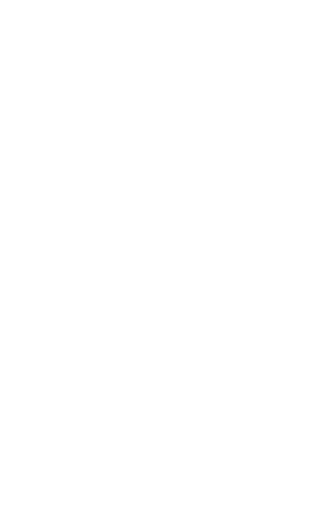
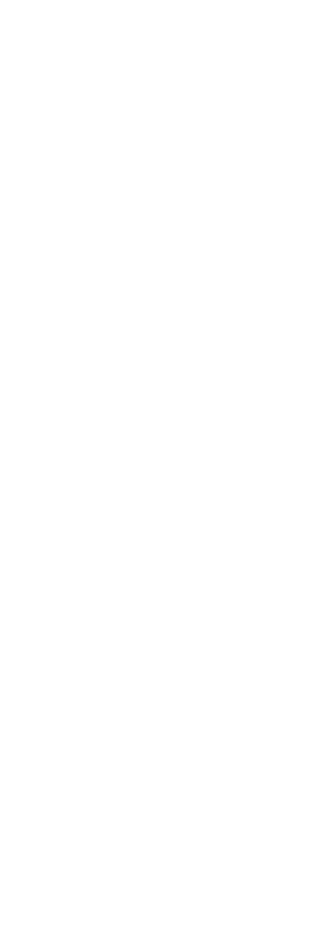

.

$x^{2}$

(n)

$x^{2}$ 


\section{Interference Effects \\ High Int $>$ Low Int $>$ CR \\ Common to Groups}

Left mid-ventrolateral PFC (BA 44/45)

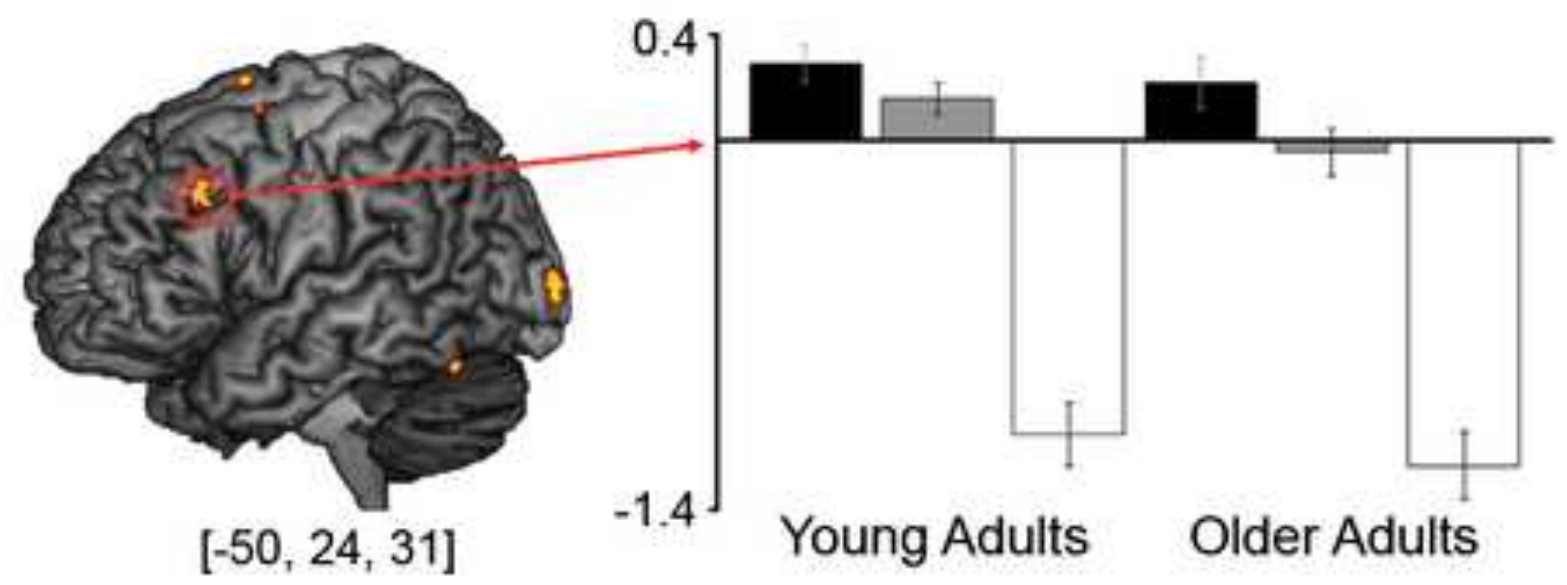

Left Superior Parietal Cortex (BA 40)

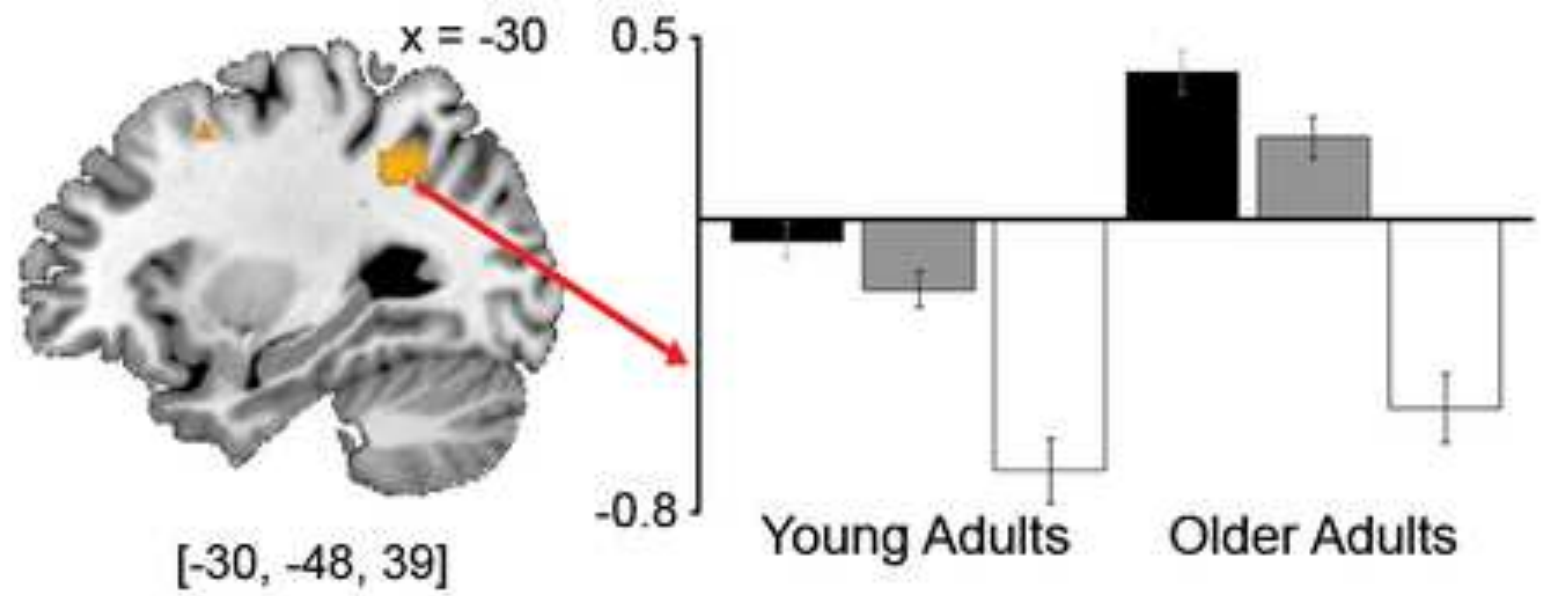

$=$ High Interference Associative Correct

$\square=$ Low Interference Associative Correct

$\square=$ Correct Rejections 


\section{Associative Memory Accuracy Effects Across Groups High Interference Correct > Incorrect}

Common to Groups

Left Anterior Hippocampus (BA 34)

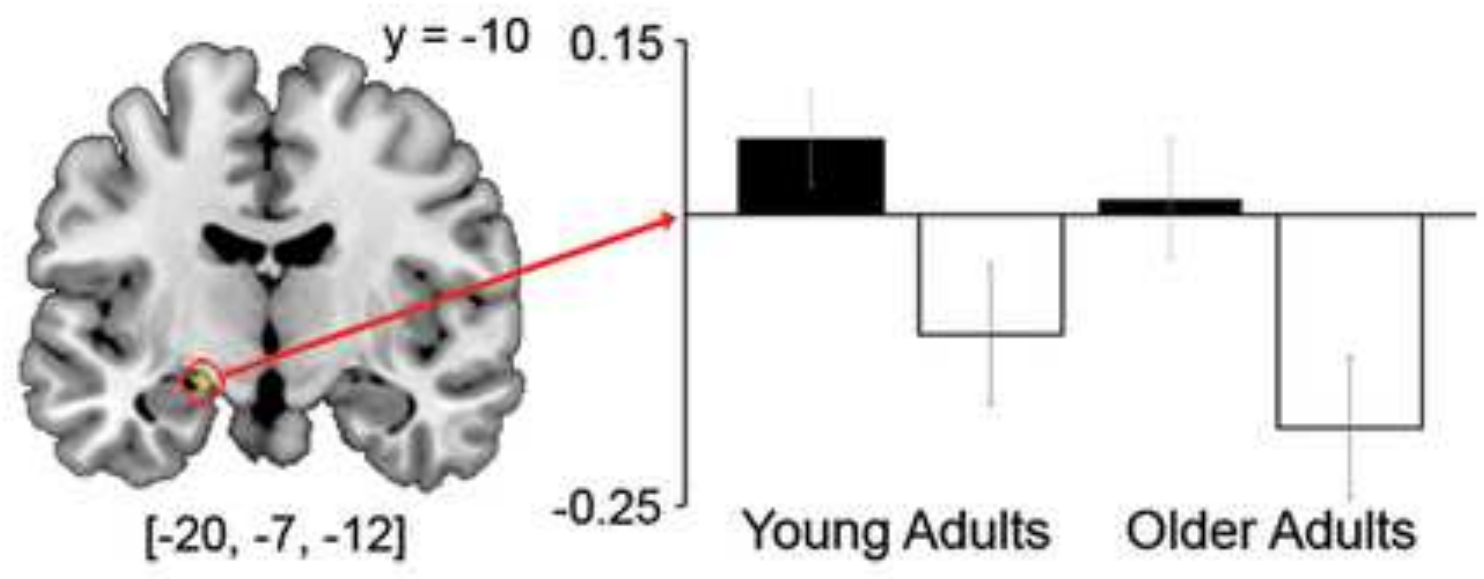

$$
\begin{aligned}
& \square=\text { High Interference Associative Correct } \\
& \square=\text { High Interference Associative Incorrect }
\end{aligned}
$$




\section{Age-Related Associative Memory Accuracy Effects High Interference Correct > Incorrect Young $>$ Old}

Left Dorsolateral Prefrontal Cortex (BA 9)

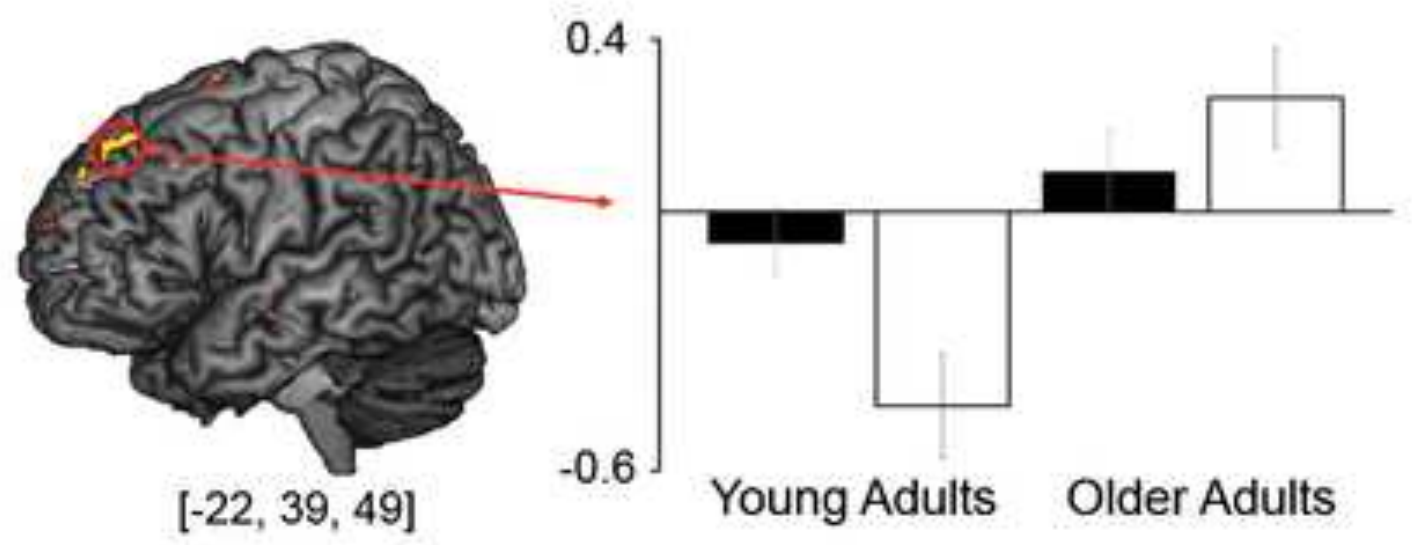

Left Anterior Prefrontal Cortex (BA 10)

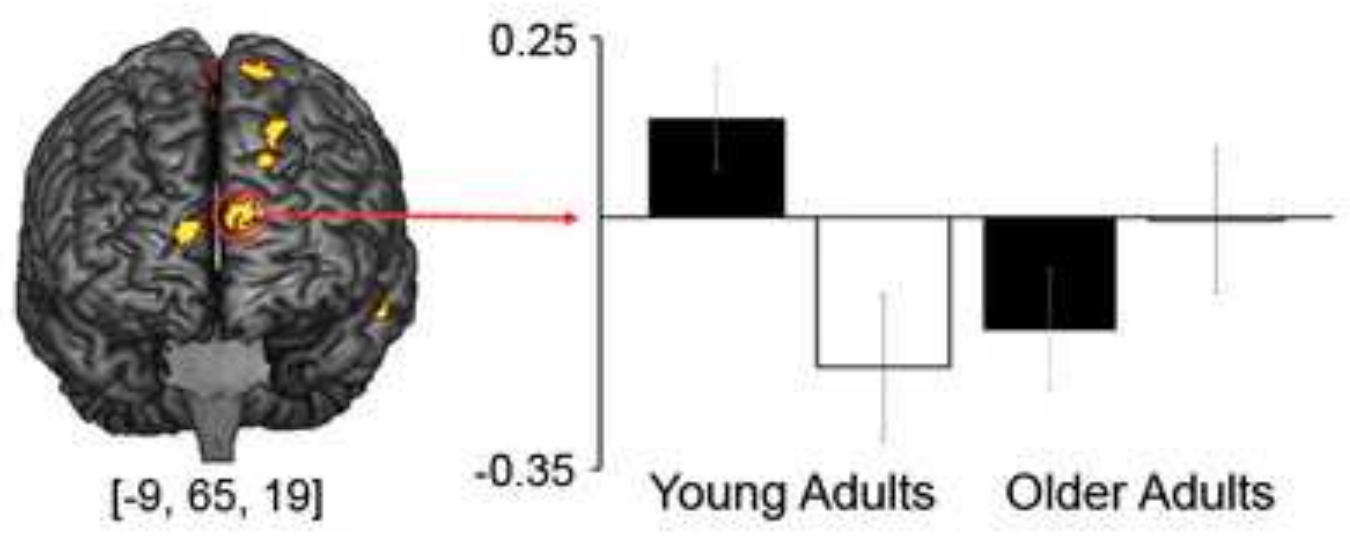

= High Interference Associative Correct

$\square=$ High Interference Associative Incorrect 


\section{Left VLPFC Sensitive to Interference, not Accuracy Common to Groups}

Left mid-ventrolateral PFC (BA 44/45)

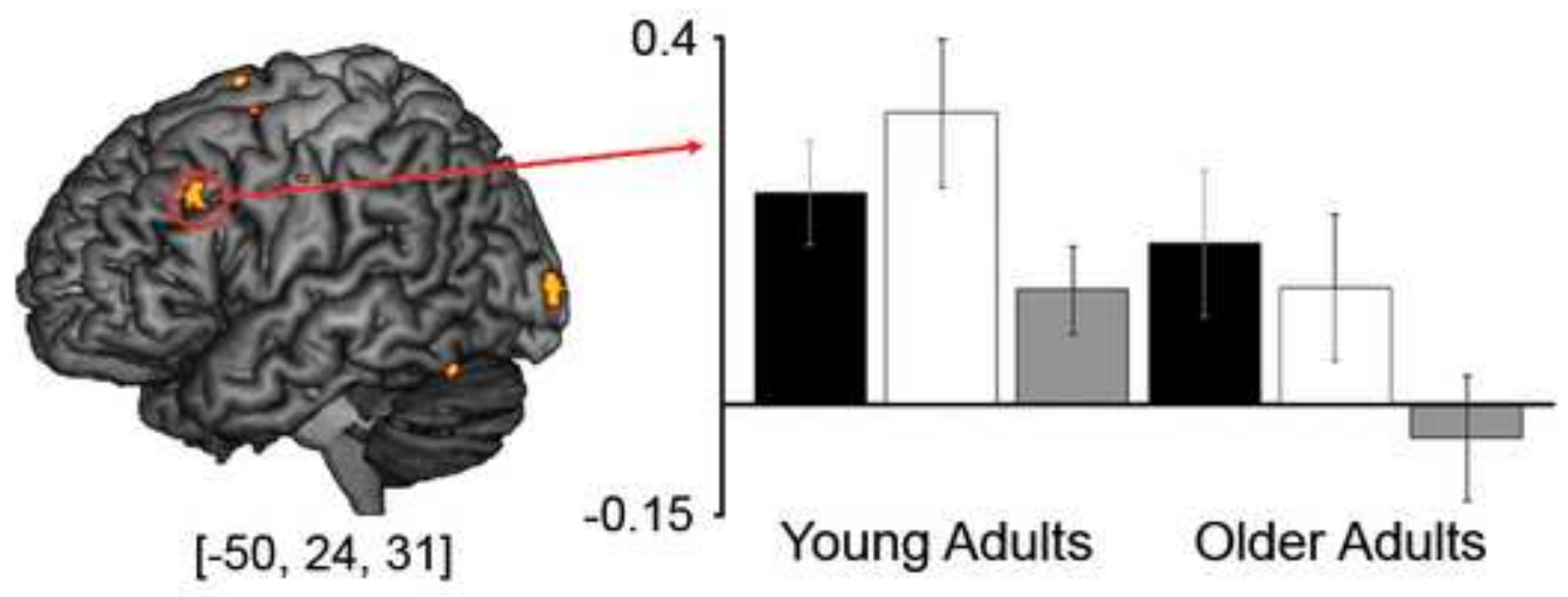

$\square=$ High Interference Associative Correct

$\square=$ High Interference Associative Incorrect $\square=$ Low Interference Associative Correct 